\title{
A perturbative expansion scheme for supermembrane and matrix theory
}

\author{
Olaf Lechtenfeld ${ }^{a}$ and Hermann Nicolai ${ }^{b}$ \\ ${ }^{a}$ Institut für Theoretische Physik and Riemann Center for Geometry and Physics, \\ Leibniz Universität Hannover, \\ Appelstrasse 2, 30167 Hannover, Germany \\ ${ }^{b}$ Max-Planck-Institut für Gravitationsphysik (Albert-Einstein-Institut), \\ Am Mühlenberg 1, 14476 Potsdam, Germany \\ E-mail: olaf.lechtenfeld@itp.uni-hannover.de, nicolai@aei.mpg.de
}

ABSTRACT: We reconsider the supermembrane in a Minkowski background and in the light-cone gauge as a one-dimensional gauge theory of area preserving diffeomorphisms (APDs). Keeping the membrane tension $T$ as an independent parameter we show that $T$ is proportional to the gauge coupling $g$ of this gauge theory, such that the small (large) tension limit of the supermembrane corresponds to the weak (strong) coupling limit of the APD gauge theory and its $\mathrm{SU}(N)$ matrix model approximation. A perturbative linearization of the supersymmetric theory suitable for a quantum mechanical path-integral treatment can be achieved by formulating a Nicolai map for the matrix model, which we work out explicitly to $\mathcal{O}\left(g^{4}\right)$. The corresponding formulæ remain well-defined in the limit $N \rightarrow \infty$; this result relies on a cancellation of infinities not present for the bosonic membrane, indicating that the $N \rightarrow \infty$ limit does not exist for the purely bosonic matrix model. Furthermore we show that the map has improved convergence properties in comparison with the usual perturbative expansions because its Jacobian admits an expansion in $g$ with a non-zero radius of convergence. Possible implications for unsolved issues with the matrix model of M theory are also mentioned.

Keywords: M(atrix) Theories, Supersymmetric Gauge Theory, Field Theories in Higher Dimensions

ARXIV EPRINT: 2109.00346 


\section{Contents}

1 Introduction and summary 1

2 Supermembrane basics 4

2.1 Supermembrane in the light-cone gauge with variable tension 4

$\begin{array}{lll}2.2 & \text { APD gauge theory and matrix model } & 7\end{array}$

$\begin{array}{ll}2.3 & \text { Setting up the path integral }\end{array}$

2.4 Physical correlators 11

3 The map to fourth order $\quad \mathbf{1 2}$

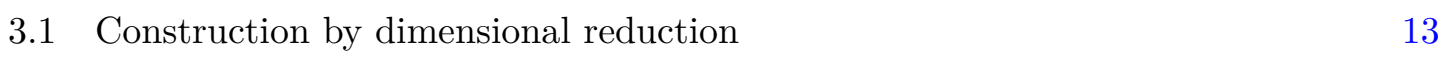

$\begin{array}{ll}3.2 & \text { Construction in the matrix model } \\ & 15\end{array}$

$\begin{array}{ll}\text { 3.3 The map to third and fourth order } & 17\end{array}$

4 The Jacobian has a non-zero radius of convergence 20

5 Outlook 22

A Tests $\quad 22$

A.1 Free action test $\quad 22$

A.2 Determinant matching test 23

\section{Introduction and summary}

The maximally supersymmetric supermembrane theory in spacetime dimension $D=11[1,2]$ is a model 'beyond' string theory that also incorporates $D=11$ supergravity [3], and is thus a candidate theory for a non-perturbative formulation of superstring theory (besides there are three more classically consistent supermembrane theories for target-space dimensions $D=4,5,7[2]$ ). As shown long ago [4] the supermembrane in a flat (Minkowski) background and in the light-cone gauge can be reformulated as a one-dimensional maximally supersymmetric gauge theory of area-preserving diffeomorphisms (APDs). Building on earlier results of [5-7] it has been shown that this model can be equivalently obtained as the $N \rightarrow \infty$ limit of a maximally supersymmetric $\mathrm{SU}(N)$ matrix model [4]. Much later the very same model was re-interpreted in terms of D0 particle quantum mechanics [8], and proposed as a model of $\mathrm{M}$ theory in [9]. For reviews of supermembrane theory with many further references, see e.g. [10-12].

The main unsolved problem of (super-)membrane theory is its quantization. Unlike for string theory there exists no gauge which linearizes the equations of motion such that the determination of quantum correlators can be effectively reduced to free field 
theory computations. Likewise, in view of the non-linearities a covariant path-integral approach à la Polyakov appears hopeless for either the bosonic or the supersymmetric membrane. A more realistically feasible approach is based on (target-space) light-cone gauge quantization. Nevertheless, even with this preferred gauge choice the solution to the problem of quantization has so far remained elusive not only because one has to deal with a fully interacting theory on the world volume, but also because it is not obvious how to set up a perturbative expansion for the quantized supermembrane. These difficulties are mirrored by corresponding difficulties of the supersymmetric $\mathrm{SU}(N)$ matrix model for $N<\infty$, as a consequence of which key issues remain unresolved to this day. Apart from questions regarding the existence and properties of the $N \rightarrow \infty$ limit for the quantized theory, there are two main issues. One concerns the target-space Lorentz invariance of the quantized supermembrane (or the matrix model in the $N \rightarrow \infty$ limit). For the classical theory and for finite $N$, Lorentz invariance is in fact violated, but can be recovered in the $N \rightarrow \infty$ limit $[13,14]$. However, there has been almost no progress on the quantized theory, which would first of all require a proper definition of the quantized Lorentz generators and ensuring their quantum consistency, before actually checking the Lorentz algebra. Amongst other things this involves the correct definition of the light-cone target-space coordinate $X^{-}$ (which matrix theory by itself 'does not know about') as a quantum operator. Consequently, it also remains an open question whether $D_{\text {crit }}=11$ is indeed the critical dimension for the supermembrane, eliminating the other classically consistent theories (see however [15] for some early results in this direction), unlike for the superstring where the well-known result $D_{\text {crit }}=10$ can be established in more than one way.

A second key issue arises in connection with correlators and scattering amplitudes for the putative massless supermembrane excitations corresponding to the graviton, the gravitino and the 3 -form field of $D=11$ supergravity. In particular, there is no (super)membrane analog known of the Veneziano and Virasoro-Shapiro amplitudes, as this would almost certainly involve higher order and non-perturbative contributions beyond the reach of conventional string technology. Remarkably, there do exist classical candidate expressions for vertex operators associated to these states [16], but like for the Lorentz generators, it has not been possible so far to turn them into well-defined quantum objects. However, even if one could compute $n$-point correlators in supermembrane theory with these vertex operators, there remains the more conceptual question as to their possible physical interpretation, and how such correlators would transcend the one-particle interpretation of first quantized superstring theory and capture non-perturbative information.

In this paper we wish to tackle the quantization of the supermembrane from a new and different perspective (for a complementary approach to quantizing supermembrane theory, see [17] and references therein). A main ingredient here is the fact that the supersymmetric APD gauge theory (alias the supersymmetric $\mathrm{SU}(\infty)$ matrix model) is the supermembrane. Our analysis leads us to the conclusion that the membrane tension $T$, made dimensionless, must be identified with the gauge coupling $g$ of the APD gauge theory. This insight allows us to set up a systematic expansion scheme in terms of a path-integral formulation where the small (large) tension limit of the membrane theory corresponds to the weak (strong) coupling limit of the APD gauge theory - a result to be contrasted 
with the somewhat murky state of affairs with the zero-tension limit of string theory. This expansion is introduced by means of a Nicolai map [18, 19] (designated by $\mathcal{T}_{g}$ ) which we first construct for the finite- $N$ theory up to and including quartic order $\mathcal{O}\left(g^{4}\right) .{ }^{1}$ Our derivation is based on a systematic procedure that relies on very recent progress in perturbatively evaluating this map for supersymmetric Yang-Mills theories in higher dimensions, see especially $[20,21]$. This prescription in principle allows for the determination of the map to any desired order. It then turns out that the pertinent formulæ all remain well-defined in the limit $N \rightarrow \infty$, via the straightforward replacement of $\mathrm{SU}(N)$ commutators by APD brackets, see especially (3.36). An accompanying $N$-dependent divergence cancels only for the supermembrane, indicating that the $N \rightarrow \infty$ limit does not exist for the bosonic matrix model $^{2}$ ('non-renormalizability' of the bosonic membrane). In arriving at this conclusion, the map $\mathcal{T}_{g}$ plays a crucial role: as far as we are aware, there is no other approach that addresses the issue of the $N \rightarrow \infty$ limit in such a direct manner. These are two main results of this paper which should eventually permit setting up an approximation scheme also for correlators and other quantities of physical interest. This might enable one to sidestep the finite- $N$ approximation altogether and to deal directly with the limiting theory for $N=\infty$.

Finally, we demonstrate that the expansion of the Jacobian in powers of $g$ has a non-zero radius of convergence. This does not yet mean that the map itself has this property, but it represents strong evidence that the perturbative expansion for $\mathcal{T}_{g}$ is indeed better behaved than the usual quantum field theoretic perturbation expansions. Of course, employing this map does not alter the standard perturbative expansion of quantum correlation functions. However, it breaks it up into two stages: in the first step, the field arguments in the correlator are replaced by the ones transformed with the inverse map $\mathcal{T}_{g}^{-1}$, which is given in terms of a tree-graph expansion. In the second step, one performs a free-field correlator of those tree graphs, which finally produces loop diagrams. Therefore, the perspective is that the tree expansion of the first stage is better than asymptotic (even convergent), while the divergent field-theoretic high-order perturbative behavior gets encoded in the free-field correlators of the second stage (see [23] for a sample calculation along these lines for $D=4$ maximal super-Yang-Mills theory). Let us also note the existence of a closed-form expression for $\mathcal{T}_{g}$ in terms of a path-ordered integral, that has no analog in standard perturbative quantum field theory [24]. In particular, this formula directly reproduces the polynomial form of $\mathcal{T}_{g}$ in those cases where such a form is known to exist (such as for supersymmetric quantum mechanics), so its further exploration might well reveal unknown special properties of maximally supersymmetric matrix theory as well.

To be sure, there still remains a long way towards a full quantum treatment of the supermembrane, both on the technical and on the conceptual side. Nevertheless, the present results offer novel perspectives on this old problem and open several new avenues for future research. A particularly intriguing one concerns the fact that the map $\mathcal{T}_{g}$ transforms the interacting functional measure of the APD gauge theory to a free one which is ultralocal in the membrane coordinates. Although this complicates setting up a sensible perturbative expansion for small membrane tension, there is a remarkable analogy here with the small-

\footnotetext{
${ }^{1}$ In the temporal gauge and actually including $O\left(g^{5}\right)$ since odd orders vanish at least up to this point.

${ }^{2}$ Related difficulties with the $N \rightarrow \infty$ limit for the bosonic matrix model were already pointed out in [22].
} 
tension limit of $\mathrm{M}$ theory considered in [25], where it is the BKL limit which likewise leads to an ultralocal theory via the causal decoupling of spatial points. The latter limit has been shown to exhibit hints of the maximal-rank hyperbolic Kac-Moody algebra $\mathrm{E}_{10}$ as a fundamental symmetry. It is a fascinating challenge to explore the possible links between these a priori different versions of the small-tension limit, and thus to reconcile two very different perspectives on $M$ theory.

The structure of this paper is as follows. In section 2 we review basic results on the light-cone gauge formulation of the supermembrane, mostly following the exposition in [4], and set up the path integral in subsection 2.3. Section 3 explains the construction of the Nicolai map both for the matrix theory and the APD gauge theory. This section contains our main result, namely an explicit form of the map $\mathcal{T}_{g}$ in an expansion to quartic order in the coupling $g$. The question of the behavior of the map in the complex $g$ plane (and thus the convergence properties of the expansion) is addressed in section 4. Finally an appendix provides details of two consistency checks on the main result derived in this paper.

\section{Supermembrane basics}

In the section we closely follow [4] to which we refer for further details of the derivation. The main difference is that we here keep the membrane tension $T$ as an independent parameter, in order to expose the link with weakly and strongly coupled Yang-Mills theory.

\subsection{Supermembrane in the light-cone gauge with variable tension}

Classically consistent supermembranes exist for target-space dimensions $D=4,5,7$ and 11 , but in the remainder we will restrict attention mostly to the maximally supersymmetric case, for which $D=11$. The target superspace coordinates $\left\{X^{\mu}, \theta\right\} \equiv\left\{X^{\mu}\left(\xi^{i}\right), \theta\left(\xi^{i}\right)\right\}$ with the range $\mu, \nu, \ldots=0,1, \ldots, D-1=10$ are then functions of the membrane world-volume coordinates

$$
\left(\xi^{i}\right) \equiv(\tau, \boldsymbol{\sigma}) \equiv\left(\tau, \sigma^{r}\right) \quad \text { where } \quad i, j, \ldots=0,1,2 \quad \text { and } \quad r, s, \ldots=1,2
$$

$\theta(\tau, \boldsymbol{\sigma})$ is a real 32-component Majorana spinor of $\mathrm{SO}(1,10)$ (we usually do not write out spinor indices). The target-space vielbein is

$$
E_{i}^{\mu}=\partial_{i} X^{\mu}+\bar{\theta} \Gamma^{\mu} \partial_{i} \theta
$$

For $D=11$, the real 32-by-32 $\Gamma$-matrices generate the $\mathrm{SO}(1,10)$ Clifford algebra, $\left\{\Gamma^{\mu}, \Gamma^{\nu}\right\}=$ $2 \eta^{\mu \nu}$. The world-volume metric is

$$
\mathrm{g}_{i j} \equiv E_{i}{ }^{\mu} E_{j}{ }^{\nu} \eta_{\mu \nu}
$$

For the light-cone gauge we split the target-space coordinates as

$$
\left\{X^{\mu}\right\} \equiv\left\{X^{+}, X^{-}, X^{a}\right\} \quad \text { with } \quad X^{ \pm}=\frac{1}{\sqrt{2}}\left(X^{10} \pm X^{0}\right) \quad \text { and } \quad\left\{X^{a}\right\} \equiv \mathbf{X}
$$


being the transverse components $(a, b, \ldots=1, \ldots, 9)$. We adopt the target-space light-cone gauge

$$
X^{+}(\tau, \boldsymbol{\sigma})=X_{0}^{+}+\tau, \quad \Gamma_{+} \theta(\tau, \boldsymbol{\sigma})=0
$$

thus identifying the target-space light-cone coordinate $X^{+}$with the world-volume time coordinate $\tau$. With these gauge choices the induced metric on the three-dimensional world volume is

$$
\begin{aligned}
& \mathrm{g}_{r s} \equiv \overline{\mathrm{g}}_{r s}=\partial_{r} \mathbf{X} \cdot \partial_{s} \mathbf{X} \\
& \mathrm{g}_{0 r} \equiv u_{r}=\partial_{r} X^{-}+\partial_{0} \mathbf{X} \cdot \partial_{r} \mathbf{X}+\bar{\theta} \Gamma_{-} \partial_{r} \theta \\
& \mathrm{g}_{00}=2 \partial_{0} X^{-}+\left(\partial_{0} \mathbf{X}\right)^{2}+2 \bar{\theta} \Gamma_{-} \partial_{0} \theta
\end{aligned}
$$

The metric determinant is

$$
\mathrm{g} \equiv \operatorname{det} \mathrm{g}_{i j}=-\Delta \overline{\mathrm{g}}
$$

with

$$
\overline{\mathrm{g}} \equiv \operatorname{det} \overline{\mathrm{g}}_{r s} \quad \text { and } \quad \Delta=-\mathrm{g}_{00}+u_{r} \overline{\mathrm{g}}^{r s} u_{s}, \quad \overline{\mathrm{g}}^{r s} \overline{\mathrm{g}}_{s t}=\delta_{t}^{r} .
$$

The supermembrane Lagrangian then becomes

$$
\mathcal{L}=T\left(-\sqrt{\overline{\mathrm{g}} \Delta}+\epsilon^{r s} \partial_{r} X^{a} \bar{\theta} \Gamma_{-} \Gamma_{a} \partial_{s} \theta\right),
$$

where we now include the membrane tension $T$ as an independent parameter. In principle the membrane tension is of dimension $[\text { mass }]^{3}$, but we here find it convenient to render all variables dimensionless by rescaling them with appropriate powers of some reference mass scale (as was already implicitly assumed in (2.5)). This reference scale has no physical meaning in and by itself, as a proper identification of the gravitational coupling (Newton constant or Planck mass) and evaluating its relation to $T$ will require the evaluation of a graviton scattering amplitude, as is the case in string theory. However, for the doubly dimensionally reduced supermembrane [26] such a relation can indeed be established by noting that $T R_{10}=\left(\alpha^{\prime}\right)^{-1}$, where $R_{10}$ is the radius of the compactified 11th dimension. Because the latter is related to the string coupling by $R_{10}=g_{s}^{2 / 3}[27]$ (see also [28]), we see that

$$
T=g_{s}^{-2 / 3}\left(\alpha^{\prime}\right)^{-1} \text {. }
$$

In this way the parameter $T$ ties together the two key parameters of string theory, and thus also with the APD gauge coupling via (2.23) below.

With these conventions the (dimensionless) canonical momenta are

$$
\begin{aligned}
P^{+} & =T \sqrt{\frac{\bar{g}}{\Delta}} \\
\mathbf{P} & =\frac{\delta \mathcal{L}}{\delta \partial_{0} \mathbf{X}}=T \sqrt{\frac{\bar{g}}{\Delta}}\left(\partial_{0} \mathbf{X}-u_{r} \mathrm{~g}^{r s} \partial_{s} \mathbf{X}\right) \equiv P^{+}\left(\partial_{0} \mathbf{X}-u_{r} \mathbf{g}^{r s} \partial_{s} \mathbf{X}\right), \\
S & =\frac{\delta \mathcal{L}}{\delta \partial_{0} \bar{\theta}}=-T \sqrt{\frac{\bar{g}}{\Delta}} \Gamma_{-} \theta \equiv-P^{+} \Gamma_{-} \theta .
\end{aligned}
$$

The last formula implies a second-class constraint (entailing the replacement of Poisson brackets by Dirac brackets). The formulæ (2.11) imply the first-class constraint

$$
\phi_{r}=\mathbf{P} \cdot \partial_{r} \mathbf{X}+P^{+} \partial_{r} X^{-}+\bar{S} \partial_{r} \theta \approx 0,
$$


which generates spatial diffeomorphisms on the membrane. This gauge freedom can be exploited to set $u^{r}=0$ in (2.6), which in turn implies

$$
\partial_{r} X^{-}=-\partial_{0} \mathbf{X} \cdot \partial_{r} \mathbf{X}-\bar{\theta} \Gamma_{-} \partial_{r} \theta
$$

To be able to solve this equation for $X^{-}$we must impose the integrability constraint

$$
\phi \equiv \epsilon^{r s}\left(\partial_{r} \partial_{0} \mathbf{X} \cdot \partial_{s} \mathbf{X}+\partial_{r} \bar{\theta} \Gamma_{-} \partial_{s} \theta\right) \approx 0
$$

This constraint generates APDs on the membrane: while general (spatial) diffeomorphisms on the membrane are generated by vector fields $\delta \xi^{r}(\boldsymbol{\sigma})$, APDs are generated by divergencefree vector fields obeying $\partial_{r}\left(\sqrt{w} \delta \xi^{r}\right)=0$ (where the reference density $\sqrt{w(\boldsymbol{\sigma})}$ coincides with the one introduced in (2.16) below). The latter are locally of the form $\sqrt{w} \delta \xi^{r}=\epsilon^{r s} \partial_{s} \delta \xi$ with a scalar parameter $\delta \xi(\boldsymbol{\sigma})$. On higher-genus membranes there are in addition topologically non-trivial diffeomeophisms formally generated by harmonic vector fields [13], which we will, however, disregard here.

With these gauge choices the (dimensionless) Hamiltonian density becomes (see also [29])

$$
\begin{aligned}
\mathcal{H}(\boldsymbol{\sigma}) & \equiv-P^{-}(\boldsymbol{\sigma})=\mathbf{P} \cdot \partial_{0} \mathbf{X}+P^{+} \partial_{0} X^{-}+\bar{S} \partial_{0} \theta-\mathcal{L} \\
& =\frac{\mathbf{P}^{2}+T^{2} \overline{\mathrm{g}}}{2 P^{+}}-T \epsilon^{r s} \partial_{r} X^{a} \bar{\theta} \Gamma_{-} \Gamma_{a} \partial_{s} \theta
\end{aligned}
$$

whose bosonic part was already derived long ago in [5-7] (for $T=1$ ). Here we see why we must choose the membrane tension to be positive; flipping the sign of $T$ will change the sign of the kinetic part of the Hamiltonian by (2.11), hence result in an instability. This is, of course, in accord with expectations.

Because $P^{+}(\tau, \boldsymbol{\sigma})$ obeys the Hamiltonian equation of motion $\partial_{\tau} P^{+}(\tau, \boldsymbol{\sigma})=0$ and transforms as a density we can set [4]

$$
P^{+}(\tau, \boldsymbol{\sigma})=P_{0}^{+} \sqrt{w(\boldsymbol{\sigma})}
$$

where $P_{0}^{+}>0$ is constant, and $\sqrt{w(\boldsymbol{\sigma})}>0$ is a reference density normalized to $\int \mathrm{d}^{2} \sigma \sqrt{w(\boldsymbol{\sigma})}=1$ (with an associated reference metric $w_{r s}(\boldsymbol{\sigma})$ on the membrane, which is however only needed when discussing target-space Lorentz invariance [13]). This leads to the (dimensionless) mass operator

$$
\mathcal{M}^{2}=-2 P_{0}^{+} P_{0}^{-}-\mathbf{P}_{0}^{2}=\int \mathrm{d}^{2} \sigma\left(\left[\mathbf{P}^{2}\right]^{\prime}+T^{2} \overline{\mathrm{g}}-2 T \epsilon^{r s} \partial_{r} X^{a} \bar{\Theta} \Gamma_{-} \Gamma_{a} \partial_{s} \Theta\right)
$$

with $P_{0}^{-}=\int \mathrm{d}^{2} \sigma P^{-}(\boldsymbol{\sigma})$ and rescaled fermionic variables ${ }^{3}$

$$
\Theta(\boldsymbol{\sigma}) \equiv \sqrt{P_{0}^{+}} \theta(\boldsymbol{\sigma})
$$

The prime in (2.17) indicates that zero modes have been removed from $\int \mathrm{d}^{2} \sigma \mathbf{P}^{2}(\boldsymbol{\sigma})$.

\footnotetext{
${ }^{3}$ Which obey the canonical (Dirac) brackets $\left\{\Theta(\boldsymbol{\sigma}), \bar{\Theta}\left(\boldsymbol{\sigma}^{\prime}\right)\right\}_{\mathrm{DB}}=(4 \sqrt{w(\boldsymbol{\sigma})})^{-1} \Gamma_{+} \delta^{(2)}\left(\boldsymbol{\sigma}, \boldsymbol{\sigma}^{\prime}\right)[4]$.
} 
Because the zero modes $X_{0}^{a}$ and $\theta_{0}$ decouple from the Lagrangian (2.9), the eigenstates of the mass operator take the form of a direct product of the massless $D=11$ multiplet $(\mathbf{4 4} \oplus \mathbf{8 4}$ bosonic and $\mathbf{1 2 8}$ fermionic states) with an eigenstate of the mass operator (2.17) [4]. For the uncompactified supermembrane the spectrum of the latter is known to be continuous [30, 31] (but becomes discrete after compactification with winding [32]). This fact can be interpreted as evidence that the supermembrane does not admit a first-quantized formulation, but must be regarded as a non-perturbative theory from the outset [11].

Finally we note that the fulfilment of the constraint (2.14) allows us to solve for the target-space coordinate $X^{-}$: we have

$$
X^{-}(\tau, \boldsymbol{\sigma})=-\int \mathrm{d}^{2} \sigma^{\prime} G^{r}\left(\boldsymbol{\sigma}, \boldsymbol{\sigma}^{\prime}\right)\left(\partial_{0} \mathbf{X} \cdot \partial_{r} \mathbf{X}\left(\tau, \boldsymbol{\sigma}^{\prime}\right)-\bar{\theta} \Gamma_{-} \partial_{r} \theta\left(\tau, \boldsymbol{\sigma}^{\prime}\right)\right)
$$

with a suitable Green's function obeying $\partial_{r} G^{r}\left(\boldsymbol{\sigma}, \boldsymbol{\sigma}^{\prime}\right)=\delta\left(\boldsymbol{\sigma}, \boldsymbol{\sigma}^{\prime}\right)[5,13]$. This formula is needed for the target-space boost generators and for the verification of target-space Lorentz invariance in the classical limit [13, 14]. It is worth pointing out that this information is not available in the matrix model as such, where the Lorentz boost generators must either be "guessed" or deduced from the supermembrane matrix-model correspondence, as in [13].

\subsection{APD gauge theory and matrix model}

With the above formula for the mass operator the supermembrane theory can be reformulated as a one-dimensional supersymmetric gauge theory of area preserving diffeomorphisms [4]. This can be seen by exploiting the algebraic identity

$$
\overline{\mathrm{g}}=\operatorname{det}\left(\partial_{r} \mathbf{X} \cdot \partial_{s} \mathbf{X}\right)=\left\{X^{a}, X^{b}\right\}\left\{X^{a}, X^{b}\right\},
$$

where the APD bracket of any two functions $A(\boldsymbol{\sigma})$ and $B(\boldsymbol{\sigma})$ on the membrane is defined by

$$
\{A(\boldsymbol{\sigma}), B(\boldsymbol{\sigma})\}:=\frac{1}{\sqrt{w(\boldsymbol{\sigma})}} \epsilon^{r s} \partial_{r} A(\boldsymbol{\sigma}) \partial_{s} B(\boldsymbol{\sigma}) .
$$

This is indeed a Lie bracket (obeying antisymmetry and the Jacobi identity) [5-7].

Then $(2.15)$ can be equivalently obtained from the supersymmetric Lagrangian ${ }^{4}$

$$
\frac{1}{\sqrt{w}} \mathcal{L}=\frac{1}{2}\left(D_{t} \mathbf{X}\right)^{2}+\bar{\Theta} \Gamma_{-} D_{t} \Theta-\frac{1}{4} g^{2}\left\{X^{a}, X^{b}\right\}^{2}+g \bar{\Theta} \Gamma_{-} \Gamma_{a}\left\{X^{a}, \Theta\right\}
$$

if we identify

$$
T=\frac{1}{2} g \text {. }
$$

In view of our comments after (2.15) we must, however, restrict this identification to positive values of $T$ and $g$, even though there appear to be no obstructions to continuing the APD gauge theory to negative couplings. Hence the small (large) tension limit of the supermembrane corresponds to the weak (strong) coupling limit of the supersymmetric APD gauge theory. The APD covariant derivative is given by

$$
D_{t} f(t, \boldsymbol{\sigma}):=\partial_{t} f(t, \boldsymbol{\sigma})+g\{\omega(t, \boldsymbol{\sigma}), f(t, \boldsymbol{\sigma})\}
$$

\footnotetext{
${ }^{4}$ While $\tau$ is the time coordinate on the membrane world-volume, we denote the Yang-Mills time coordinate by $t$, but keep the erstwhile membrane coordinates $\sigma^{r}$ as labels for the APD gauge group.
} 
with the APD gauge field $\omega(t, \boldsymbol{\sigma})$ which is here introduced ad hoc, as it is absent from the supermembrane action. The Lagrangian (2.22) is nothing but the dimensional reduction of maximally extended super-Yang-Mills theory [33] to one (time) dimension, with the identifications $\omega \equiv A_{0}$ and $X_{a} \equiv A_{a}$, and $g$ the usual Yang-Mills coupling, but now with the infinite-dimensional APD gauge group. This works precisely in the dimensions where pure supersymmetric Yang-Mills theories exist, namely $D=3,4,6,10$ [33], in agreement with the admissible target-space dimensions 4,5,7 and 11 for supermembranes.

The group of (homotopically trivial) APDs on the membrane can be approximated by the finite-dimensional unitary groups $\mathrm{SU}(N)$, such that the full group is recovered in the limit $N \rightarrow \infty[5-7]$. Replacing APDs by $\mathrm{SU}(N)$ gives the matrix model of $\mathrm{M}$ theory. For this approximation one expands all functions on the membrane into a complete orthonormal set of functions $Y^{A}(\boldsymbol{\sigma})$,

$$
\int \mathrm{d}^{2} \sigma \sqrt{w(\boldsymbol{\sigma})} Y^{A}(\boldsymbol{\sigma}) Y^{B}(\boldsymbol{\sigma})=\delta^{A B},
$$

where we separate off the zero modes,

$$
\begin{gathered}
X_{a}(t, \boldsymbol{\sigma})=X_{a}^{(0)}(t)+\sum_{A=1}^{\infty} X_{a}^{A}(t) Y^{A}(\boldsymbol{\sigma}), \\
\omega(t, \boldsymbol{\sigma})=\omega^{(0)}(t)+\sum_{A=1}^{\infty} \omega^{A}(t) Y^{A}(\boldsymbol{\sigma}) \\
\Theta(t, \boldsymbol{\sigma})=\Theta^{(0)}(t)+\sum_{A=1}^{\infty} \Theta^{A}(t) Y^{A}(\boldsymbol{\sigma}) .
\end{gathered}
$$

The zero modes $X_{a}^{(0)}(t)$ and $\Theta^{(0)}(t)$ decouple in $(2.22)$, where $X_{a}^{(0)}(t)$ describes the center of mass motion of the membrane as a whole. Likewise, the gauge zero mode $\omega^{(0)}(t)$ drops out in the Lagrangian (as it acts effectively like a U(1) gauge field, which cannot couple because both $X_{a}^{(0)}$ and $\Theta^{(0)}$ are real). The remaining non-zero modes describe the 'internal' degrees of freedom of the supermembrane. The APD gauge group can thus be approximated by $\mathrm{SU}(N)$, as is most easily and explicitly done for $S^{2}[5-7]$ and $T^{2}[13,34,35]$, by cutting off the mode expansions at $N^{2}-1$ (ignoring topological modes) and replacing the APD-brackets by $\mathrm{SU}(N)$ commutators. In fact, as shown in [36] the $\mathrm{SU}(N)$ approximation works for any genus of the membrane. Consequently, we have

$$
f_{\mathrm{APD}}^{A B C} \equiv \int d^{2} \sigma \sqrt{w(\boldsymbol{\sigma})} Y^{A}(\boldsymbol{\sigma})\left\{Y^{B}(\boldsymbol{\sigma}), Y^{C}(\boldsymbol{\sigma})\right\}=\lim _{N \rightarrow \infty} f^{A B C}(N)
$$

with $\mathrm{SU}(N)$ structure constants $f^{A B C}(N)$. Hence the expansion labels $A, B, \ldots=1, \ldots, N^{2}-1$ are thus turned into Yang-Mills indices, while $a, b, \ldots$ are transverse (for membrane) and space-like (for supersymmetric Yang-Mills) indices.

After these preparations, the matrix-model Lagrangian assumes the standard form ${ }^{5}$

$$
\mathcal{L}=\frac{1}{2}\left(D_{t} X_{a}^{A}\right)^{2}-\mathrm{i} \theta_{\alpha}^{A} D_{t} \theta_{\alpha}^{A}-\frac{1}{4} g^{2}\left(f^{A B C} X_{b}^{B} X_{c}^{C}\right)^{2}-\frac{\mathrm{i}}{2} g f^{A B C} \theta_{\alpha}^{A} \gamma_{\alpha \beta}^{a} X_{a}^{B} \theta_{\beta}^{C},
$$

\footnotetext{
${ }^{5}$ For finite-dimensional gauge groups these supersymmetric matrix models were first obtained in [37-39].
} 
where we have now switched to $\operatorname{SO}(9)$ spinors $\theta_{\alpha}^{A}$ with 16 real components and where

$$
D_{t} \theta^{a}=\partial_{t} \theta^{A}+g f^{A B C} \omega^{B} \theta^{C}
$$

is the $\mathrm{SU}(N)$ covariant derivative. The real symmetric 16 -by-16 matrices $\gamma_{a}$ generate the $\mathrm{SO}(9)$ Clifford algebra, $\left\{\gamma^{a}, \gamma^{b}\right\}=2 \delta^{a b}$. Variation w.r.t. $\omega^{A}$ yields the constraint

$$
f^{A B C}\left(X_{a}^{B} D_{t} X_{a}^{C}+\theta_{\alpha}^{B} \theta_{\alpha}^{C}\right) \approx 0
$$

which is equivalent to the canonical generator of $\mathrm{SU}(N)$ gauge transformations (after performing this variation we can put $\omega^{A}=0$ everywhere). The Lagrangian (2.28) is the one that underlies the $\mathrm{M}$ theory conjecture of [9], see also [40-42].

Of course, the group of area-preserving diffeomeorphisms also depends on the topology of the membrane. For topologically non-trivial membranes, the APDs continuously connected to the identity constitute a normal subgroup $\mathrm{APD}_{0}$ within the group of all area-preserving diffeomorphisms [13]. It is only the subgroup $\mathrm{APD}_{0}$ that can be approximated by $\mathrm{SU}(N)$ (indeed for arbitrary genus of the membrane [36]), whereas diffeomorphisms in the quotient $\mathrm{APD} / \mathrm{APD}_{0}$ are beyond the reach of the $\mathrm{SU}(N)$ matrix approximation. ${ }^{6}$ The fact that one can interpolate between membranes of different topology by means of thin tubes that cost no energy (see e.g. [11] for an explanation of this point) then raises the question of how to accommodate different topologies in a single unified APD formulation, and suggests adopting a 'universal' APD group encompassing membranes of all genera, perhaps along the lines of [43].

\subsection{Setting up the path integral}

Our goal is now to set up a path-integral formulation that should eventually permit the computation of correlators of physically relevant quantities, and complement the canonical quantization methods underlying many treatments of the matrix model. We shall thus be interested in evaluating correlation functions of the type

$$
\begin{gathered}
\left\langle\mathcal{O}_{1} \cdots \mathcal{O}_{n}\right\rangle_{g}=\int \prod \mathcal{D} X_{a}(t, \boldsymbol{\sigma}) \mathcal{D} \theta_{\alpha}(t, \boldsymbol{\sigma}) \mathcal{D} \omega(t, \boldsymbol{\sigma}) \mathcal{D} C(t, \boldsymbol{\sigma}) \mathcal{D} \bar{C}(t, \boldsymbol{\sigma}) \times \\
\times \mathcal{O}_{1}[\mathbf{X}, \theta] \cdots \mathcal{O}_{n}[\mathbf{X}, \theta] \exp \left(\mathrm{i} \mathrm{S}_{\text {tot }}\right)
\end{gathered}
$$

where the precise form of the functionals $\mathcal{O}_{i}[\mathbf{X}, \theta]$ need not be specified at this point. Because this is a gauge theory, the full action

$$
\mathrm{S}_{\mathrm{tot}}=\mathrm{S}+\mathrm{S}^{\prime}
$$

with $\mathrm{S}=\int \mathrm{d} t \mathcal{L}$ must comprise a gauge-fixing part $\mathrm{S}^{\prime}=\int \mathrm{d} t \mathcal{L}^{\prime}$. For higher-dimensional Yang-Mills theories there are two preferred choices, namely the Lorenz gauge $\partial^{\mu} A_{\mu}=0$, and the axial gauge $n^{\mu} A_{\mu}=0$ (which includes the light-cone gauge for null vectors $n^{\mu}$ ). In the reduction to one time dimension the axial gauge is necessarily identical with the

\footnotetext{
${ }^{6}$ For fixed genus, the quotient $\mathrm{APD} / \mathrm{AP} \mathrm{D}_{0}$ is nothing but the mapping class group of the membrane (we are grateful to A. Kleinschmidt for a discussion on this point).
} 
temporal gauge. Consequently, we have two preferred choices for the gauge-fixing part, namely

$$
\begin{array}{ll}
\mathcal{L}^{\prime}=-\frac{1}{2 \xi}\left(\partial_{t} \omega\right)^{2}+\bar{C} \partial_{t} D_{t} C & \text { (Lorenz gauge) }, \\
\mathcal{L}^{\prime}=-\frac{1}{2 \xi} \omega^{2}+\bar{C} D_{t} C & \text { (temporal gauge) } .
\end{array}
$$

A further peculiarity of one dimension is that the temporal gauge implies the Lorenz gauge

$$
\omega(t, \boldsymbol{\sigma})=0 \quad \Rightarrow \quad \partial_{t} \omega(t, \boldsymbol{\sigma}) \equiv \dot{\omega}(t, \boldsymbol{\sigma})=0 .
$$

$C(t, \boldsymbol{\sigma})$ and $\bar{C}(t, \boldsymbol{\sigma})$ are the usual Faddeev-Popov ghosts [44, 45], and $\xi$ is a real parameter which will be eventually sent to zero to put the theory on the gauge hypersurface. After trading the $\boldsymbol{\sigma}$ dependence for $\mathrm{SU}(N)$ indices, we are left with a quantum mechanical path integral describing finitely many degrees of freedom. Because of the supersymmetry there is no need for a normalization factor in (2.31) (as can be easily checked for $g=0$ with both gauge choices). In passing we note that we can of course equivalently switch to a Euclidean formulation by flipping the sign in the kinetic terms $\left(\dot{X}_{a}\right)^{2}, \dot{\omega}^{2}$ and for the ghosts, and by replacing the oscillatory exponent by $\exp \left(-\mathrm{S}_{\text {tot }}\right)$; the factor $\mathrm{i}$ is then absent in the spinor kinetic term.

An important part of our construction is that we consider the path integral in a form where the fermions (and also the ghosts) are integrated out. For the temporal gauge and for finite $N$ the integration over $\theta_{\alpha}^{A}(t)$ results in the Matthews-Salam-Seiler (MSS) determinant $[46,47]$

$$
\Delta_{\mathrm{MSS}}[\omega=0, \mathbf{X}]=\left[\operatorname{det}\left(\delta^{A B} \delta_{\alpha \beta} \delta\left(t_{1}-t_{2}\right)+g K_{\alpha \beta}^{A B}\left(t_{1}, t_{2}\right)\right)\right]^{1 / 2}
$$

which is actually a Pfaffian because we are integrating over real fermions. The integral kernel appearing in this expression is

$$
K_{\alpha \beta}^{A B}\left(t_{1}, t_{2}\right):=\varepsilon\left(t_{1}-t_{2}\right) f^{A C B} \gamma_{\alpha \beta}^{a} X_{a}^{C}\left(t_{2}\right) .
$$

This is a real operator which is however not symmetric because hermitian conjugation also exchanges the arguments $t_{1}$ and $t_{2}$. Furthermore, we have taken out trivial factors of $\operatorname{det}\left(\partial_{t}\right)$ (which anyway cancel in the supersymmetric path integral). The free fermion propagator $\varepsilon$ is just the Green's function for $\partial_{t}$,

$$
\varepsilon\left(t-t^{\prime}\right)=\left[\partial_{t}^{-1}\right]\left(t, t^{\prime}\right)=\int \frac{\mathrm{d} p}{2 \pi} \frac{\mathrm{i} p}{p^{2}-\mathrm{i} \epsilon} \mathrm{e}^{-\mathrm{i} p\left(t-t^{\prime}\right)}=\Theta\left(t-t^{\prime}\right)-\frac{1}{2}=-\varepsilon\left(t^{\prime}-t\right) .
$$

This choice of integration constant implies $\varepsilon(0)=0$ as well as

$$
\int \mathrm{d} t \varepsilon\left(t-t^{\prime}\right)=0
$$

Our particular choice is important for the tests in the appendix which otherwise cannot be satisfied (it is also consistent with the dimensional reduction of the usual Dirac propagator). In section 4 we will study some properties of this determinant in more detail and prove in particular that the expansion of $\log \left(\Delta_{\mathrm{MSS}}\right)$ in powers of $g$ has a non-zero radius of convergence with suitable technical assumptions on the behavior of $X_{a}^{A}(t)$. We also note 
that we have no positivity statement about $\Delta_{\text {MSS }}$ (though the fermion determinant is non-negative for complex fermions!). Similarly, the determinant cannot be shown to be an even function of $g$ because of the non-vanishing $\operatorname{trace} \operatorname{tr}\left(\gamma^{a_{1}} \cdots \gamma^{a_{9}}\right)=16 \epsilon^{a_{1} \cdots a_{9}}$.

For the infinite-dimensional APD gauge group we must be a little more careful: while the kinetic term of (2.22) is local in $\sigma$, the interaction term is not because it contains derivatives in $\boldsymbol{\sigma}$. To take into account this non-locality we can formally replace the integral kernel (2.36) by

$$
K_{\alpha \beta}^{\mathrm{APD}}\left(t_{1}, t_{2} ; \boldsymbol{\sigma}_{1}, \boldsymbol{\sigma}_{2}\right):=\varepsilon\left(t_{1}-t_{2}\right) \gamma_{\alpha \beta}^{a} \frac{1}{\sqrt{w\left(\boldsymbol{\sigma}_{\mathbf{1}}\right)}} \epsilon^{r s} \frac{\partial X_{a}\left(t_{2}, \boldsymbol{\sigma}_{1}\right)}{\partial \sigma_{1}^{r}} \delta\left(\boldsymbol{\sigma}_{1}, \boldsymbol{\sigma}_{2}\right) \frac{\partial}{\partial \sigma_{2}^{s}}
$$

and the identity operator by $\delta_{\alpha \beta} \delta\left(t_{1}-t_{2}\right) \delta\left(\boldsymbol{\sigma}_{1}, \boldsymbol{\sigma}_{2}\right)$, with the proviso that folding with this kernel now also contains an integral over $\boldsymbol{\sigma}$. When expanding the logarithm of the MSS determinant using log det $=$ tr log, we encounter for each trace a divergent factor $\delta(\boldsymbol{\sigma}, \boldsymbol{\sigma})$. This factor corresponds to a factor of $N$ arising in the matrix-model regularization for each trace over the Yang-Mills indices (as in $f^{A C D} f^{B C D}=N \delta^{A B}$ ), which also diverges in the limit $N \rightarrow \infty .^{7}$ Importantly, for the supersymmetric theory this divergence is compensated by a corresponding factor from the Jacobian, as follows directly from (3.2). This cancellation explains why our final result (3.36) is perfectly well-defined. At the same time it indicates that the $N \rightarrow \infty$ limit does not exist for the purely bosonic matrix model, thus giving meaning to the statement that the 'bosonic membrane is non-renormalizable'.

\subsection{Physical correlators}

With the path integral formalism at hand we can now in principle proceed to calculate gaugevariant and gauge-invariant correlators of suitable objects. But what are the physically relevant operators $\mathcal{O}[\mathbf{X}, \theta]$ ? As in string theory, for the membrane the latter should be associated to vertex operators describing the emission or absorption of certain oneparticle excitations from the membrane. As first shown in [16] there indeed exist the classical analogs of supermembrane light-cone vertex operators exciting the massless states of the supermembrane, which comprise the massless supermultiplet of maximal $D=11$ supergravity [3]. The related expressions must satisfy various consistency constraints (target-space and world-volume gauge invariance, linear and non-linear supersymmetry) which are explained at length in [16], corresponding to (but more complicated than) the ones known from type II superstring theory. In particular, in analogy with closed-string vertex operators they are to be integrated over the membrane world volume. For instance, for the transverse graviton components we have [16]

$$
\mathcal{O}[\mathbf{X}, \theta]=\int \mathrm{d} t \mathrm{~d} \boldsymbol{\sigma} V_{h}[\mathbf{X}, \theta]
$$

with

$$
\begin{aligned}
V_{h}[\mathbf{X}, \theta]= & h_{a b}\left[D_{t} X^{a} D_{t} X^{b}-\left\{X^{a}, X^{c}\right\}\left\{X^{b}, X^{c}\right\}-\mathrm{i} \bar{\theta} \gamma^{a}\left\{X^{b}, \theta\right\}\right. \\
& \left.-\frac{1}{2} D_{t} X^{a} \bar{\theta} \gamma^{b c} \theta k_{c}-\frac{1}{2}\left\{X^{a}, X^{c}\right\} \bar{\theta} \gamma^{b c d} \theta k_{c}+\frac{1}{2} \bar{\theta} \gamma^{a c} \theta \bar{\theta} \gamma^{b d} \theta k_{c} k_{d}\right] \mathrm{e}^{-\mathrm{i} k \cdot \mathbf{X}+\mathrm{i} k^{-} t}
\end{aligned}
$$

\footnotetext{
${ }^{7}$ To get the proper APD structure constants in the $N \rightarrow \infty$ limit, one must adopt a suitable normalization of the $\mathrm{SU}(N)$ generators, see e.g. appendix A of [13].
} 
where $h_{a b}$ is the transverse graviton polarization tensor, and $\left\{k_{a}\right\}=\boldsymbol{k}$ denotes the transverse components of the target-space momentum. For the light-cone gauge target-space momentum $k^{\mu}$ one must furthermore assume $k^{+}=0$ in order to avoid having to deal with the light-cone coordinate $X^{-}(\tau, \boldsymbol{\sigma})$ in the exponential (as is also customary in string theory [48]). Remarkably, and unlike for superstring theory, there do not appear to exist analogs of the string vertex operators for massive string states. This would be in accord with the fact that the supermembrane is not a first quantizable (i.e. one-particle) theory [11] and for finite $N$ consistent with the D0-multiparticle interpretation of [9].

Because the light-cone vertex operators are given by complicated expressions, and because the measure in (2.31) is not Gaussian, no sustained attempt has been made, as far as we are aware, to evaluate their correlators. Neither has it been possible so far to set up a perturbative expansion, as this will also require understanding the quantum corrections (renormalizations) that are necessary for the vertex operators to remain well-defined in the quantized interacting theory. Our line of attack will therefore be a different one, in that we will reformulate the above path integral in terms of a Nicolai map. A main advantage of such an approach is that the formulæ to be presented below remain perfectly well-defined in the limit $N \rightarrow \infty$ and can thus be consistently implemented also in the APD path integral. Consequently, it may be possible in this way to sidestep the detour via the finite- $N$ matrix model, and to directly tackle the $N=\infty$ theory right away. Possible applications of this technology to supermembrane vertices will, however, be left to future work.

\section{The map to fourth order}

The method that we propose here to tackle expressions like (2.31) is based on the Nicolai map $\mathcal{T}_{g}[18,19,49-53]$, exploiting recent progress in determining this map to higher orders in $g[20,21,54,55]$. This map is a non-local and non-linear field transformation, which maps the theory to a free theory in such a way that after integrating out the fermions (gaugini and ghosts) the product of the resulting fermionic determinants equals the Jacobian of the map $\mathcal{T}_{g}$ at least locally in field space. For operators $\mathcal{O}_{k}\left(t_{k}\right)$ built from $X_{a}$ (and $\omega$ ) alone, this enables us to re-express the expectation value (2.31) in the matrix theory as a free-field correlator of transformed bosonic fields, viz.

$$
\left\langle\mathcal{O}_{1}\left(t_{1}\right) \cdots \mathcal{O}_{n}\left(t_{n}\right)\right\rangle_{g}=\left\langle\mathcal{T}_{g}^{-1}\left(\mathcal{O}_{1}\left(t_{1}\right)\right) \cdots \mathcal{T}_{g}^{-1}\left(\mathcal{O}_{n}\left(t_{n}\right)\right)\right\rangle_{0}
$$

where integrating out the gaugini and ghosts is trivial on the right-hand side because the transformed operators are purely bosonic ones. We can therefore read this relation as one in the integrated-out theory as well as in the original one including the fermions. ${ }^{8}$ A key property of the map $\mathcal{T}_{g}$ is the equality of its functional Jacobian with the product of the fermionic determinants obtained by integrating out all anticommuting variables, to wit,

$$
\operatorname{det}\left(\frac{\delta \mathcal{T}_{g} X}{\delta X}\right)=\Delta_{\mathrm{MSS}}[\omega, \mathbf{X}] \Delta_{\mathrm{FP}}[\omega, \mathbf{X}]
$$

\footnotetext{
${ }^{8}$ An extension to include fermionic or ghost arguments in $\mathcal{O}_{k}$ is straightforward but renders them nonlocal in the integrated-out theory.
} 
where $\Delta_{\mathrm{FP}}$ and $\Delta_{\text {MSS }}$ are, respectively, the Faddeev-Popov determinant $[44,45]$ and the MSS determinant $(2.35)$ [46, 47]. We refer readers to [20, 21, 24, 54, 55] for recent progress in constructing the map $\mathcal{T}_{g}$ for pure supersymmetric Yang-Mills theories in all relevant dimensions. A crucial simplification follows from (2.34), since it allows us to largely ignore the distinction between 'on-shell' and 'off-shell' $R$-prescriptions in [20, 21] that must be taken into account in more than one dimension.

Computing quantum correlation functions via (3.1) may in particular shed new light onto the combinatorial divergences appearing in higher orders of perturbation theory, due to the separation of the computation into two stages. The first step amounts to writing out the operators appearing on the right-hand side of (3.1) in powers of $g$, for which the following section provides evidence of a convergent tree-graph expansion. The second step consists of computing the free-boson correlators in (3.1), which connects the leaves of the trees in all possible ways, giving rise to the known UV divergencies and graph combinatorics. This approach thus amounts to a reorganization of the standard perturbative expansion: it removes all bosonic tadpoles and fermion loops, effectively combining them into non-standard (hybrid) loops which in higher dimensions have the supersymmetry-induced cancellation of the leading UV divergence already built in [50-53]. Therefore, although integrating out the fermions does produce a highly nonlocal, and thus seemingly more intricate, bosonic theory, thanks to the hidden supersymmetry its correlation functions are not more complicated but potentially simpler than those of the local formulation and offer new insights.

\subsection{Construction by dimensional reduction}

The goal of this section is the construction of $\mathcal{T}_{g}$ [19, 49-53] for the APD and $\mathrm{SU}(N)$ supersymmetric matrix models (2.22) and (2.28). This can be done either by repeating the construction procedure described in $[49,53]$ for this particular theory, or by dimensionally reducing the map for ten-dimensional super Yang-Mills theory to one-dimensional matrix mechanics. Let us first choose the second path.

Since we only have an on-shell formulation of supersymmetry in ten dimensions, we cannot employ the general scheme $[20,21]$ for arbitrary gauge fixing but have to stick to the Lorenz gauge, for which the map was presented on the gauge hypersurface in the critical spacetime dimensions $D=3,4,6$ and 10 , to $O\left(g^{3}\right)$ in [55] and to $O\left(g^{4}\right)$ in [20]. In the dimensional reduction all quantities loose their coordinate dependence except for a dependence on time $t$, and the $D$ components of the gauge potential become $(D-1)$ dynamical matrices $X_{a}(t)$ and one non-dynamical matrix $\omega(t)$. The Lorenz gauge reduces to $\partial_{t} \omega \equiv \dot{\omega}=0$, hence the matrix $\omega$ is a constant on the gauge hypersurface. It will turn out that it is invariant under the map $\mathcal{T}_{g}$.

Let us recall the salient facts of the construction, keeping $D$ arbitrary and denoting by $r$ the dimension of the corresponding Majorana spinor representation. The map $\mathcal{T}_{g}$ is a nonlinear and nonlocal field transformation

$$
\mathcal{T}_{g}:\left(X_{a}(t), \omega\right) \mapsto\left(X_{a}^{\prime}(t), \omega^{\prime}\right) .
$$

It affords to express the quantum correlator $\langle F\rangle_{g}$ of an arbitrary bosonic functional $F$ at gauge coupling $g$ in terms of a free correlator $(g=0)$ of the same functional, but with its 
arguments transformed by the inverse map,

$$
\langle F[X, \omega]\rangle_{g}=\left\langle F\left[\mathcal{T}_{g}^{-1} X, \mathcal{T}_{g}^{-1} \omega\right]\right\rangle_{0}
$$

An infinitesimal (in $g$ ) version reads

$$
\partial_{g}\langle F[X, \omega]\rangle_{g}=\left\langle\left(\partial_{g}+R_{g}[X, \omega]\right) F[X, \omega]\right\rangle_{g},
$$

where the "coupling flow operator" $R_{g}$ is a linear functional integro-differential operator with a nonlinear and nonlocal dependence on $X$ and $\omega$. As the construction is perturbative in the coupling $g,{ }^{9}$ we expand (note the index shift)

$$
R_{g}[X, \omega]=\sum_{k=1}^{\infty} g^{k-1} \mathrm{R}_{k}[X, \omega]=\mathrm{R}_{1}[X, \omega]+g \mathrm{R}_{2}[X, \omega]+g^{2} \mathrm{R}_{3}[X, \omega]+g^{3} \mathrm{R}_{4}[X, \omega]+\ldots
$$

Integrating the infinitesimal flow equation (3.5) yields $\mathcal{T}_{g}^{-1}$ and finally

$$
\begin{aligned}
\mathcal{T}_{g} X_{a}= & X_{a}-g \mathrm{R}_{1} X_{a}-\frac{1}{2} g^{2}\left(\mathrm{R}_{2}-\mathrm{R}_{1}^{2}\right) X_{a}-\frac{1}{6} g^{3}\left(2 \mathrm{R}_{3}-\mathrm{R}_{1} \mathrm{R}_{2}-2 \mathrm{R}_{2} \mathrm{R}_{1}+\mathrm{R}_{1}^{3}\right) X_{a} \\
& -\frac{1}{24}\left(6 \mathrm{R}^{4}-2 \mathrm{R}_{1} \mathrm{R}_{3}-3 \mathrm{R}_{2}^{2}+\mathrm{R}_{1}^{2} \mathrm{R}_{2}-6 \mathrm{R}_{3} \mathrm{R}_{1}+2 \mathrm{R}_{1} \mathrm{R}_{2} \mathrm{R}_{1}+3 \mathrm{R}_{2} \mathrm{R}_{1}^{2}-\mathrm{R}_{1}^{4}\right) X_{a}+\ldots
\end{aligned}
$$

in terms of the flow operator's expansion coefficients. We have displayed the result to $O\left(g^{4}\right)$ since we shall evaluate the map to this order, and we omitted the analogous formula for $\omega$ because it reduces to $\mathcal{T}_{g} \omega=\omega$.

In order to avoid cluttering the equations with indices, we mostly suppress spinor and color indices as well as time dependence and employ the DeWitt summation convention (suppressing also time integrals) in the remainder of this section. We find it convenient to let the flow operator act (by functional differentiation) to the left. It is then given by a variation $\frac{\overleftarrow{\delta}}{\delta X_{a}^{A}(t)}$ followed by a string of matrices in color, spinor and coordinate space, such as

$$
\left(X_{a} \times\right)^{A B}\left(t, t^{\prime}\right)=f^{A M B} X_{a}^{M}(t) \delta\left(t-t^{\prime}\right) \Rightarrow\left(X_{c} \times X_{d}\right)^{A}(t)=f^{A M N} X_{c}^{M}(t) X_{d}^{N}(t)
$$

and propagators $G$ and $S$ defined by

$$
\left[D_{t} G\right]_{\alpha \beta}^{A B}\left(t, t^{\prime}\right)=\delta^{A B} \delta_{\alpha \beta} \delta\left(t-t^{\prime}\right), \quad\left[\left(D_{t}+g \gamma^{a} X_{a} \times\right) S\right]_{\alpha \beta}^{A B}\left(t, t^{\prime}\right)=\delta^{A B} \delta_{\alpha \beta} \delta\left(t-t^{\prime}\right)
$$

with $D_{t} \equiv D_{0}=\partial_{t}+g \omega \times$. We note that $\omega \times$ and $X_{a} \times$ are to be considered as matrices in color space. The product of all these objects is to be executed in canonical fashion, where we suppress obvious unit factors in the formulæ. Observe also that $G$ is not the ghost propagator whose defining equation contains another derivative $\partial_{t}$. This is because in all relevant expressions the ghost propagator appears with a derivative $\partial_{t}$.

A careful dimensional reduction of the coupling flow operator eq. (1.19) of [55] then yields $^{10}$

$$
\overleftarrow{R}=-\frac{1}{r} \frac{\overleftarrow{\delta}}{\delta X_{a}} \operatorname{tr}\left[\left(\gamma_{a}-g X_{a} \times G\right) S\left(\frac{1}{2} \gamma^{c d} X_{c} \times X_{d}+\gamma^{d} \omega \times X_{d}\right)\right]
$$

\footnotetext{
${ }^{9}$ There exists, however, a universal nonperturbative formula for the map, see [24].

${ }^{10}$ Note that we have split $\mathcal{R}=\partial_{g}+R$.
} 
where the explicit trace refers to the spinor space, and we have dropped a term proportional to $\frac{1}{g} \dot{\omega}$. Here, the first round bracket arises from a non-abelian projector [55] which in the Lorenz gauge reads $(\mu=(t, a))$

$$
P_{\mu}^{\nu}=\delta_{\mu}^{\nu}-D_{\mu}(\partial \cdot D)^{-1} \partial^{\nu} \stackrel{\text { reduction }}{\longrightarrow} \delta_{\mu}^{\nu}-D_{\mu} D_{t}^{-1} \partial_{t}^{-1} \partial^{\nu}
$$

which obeys $\partial^{\mu} P_{\mu}^{\nu}=0=P_{\mu}{ }^{\nu} D_{\nu}$ and yields

$$
P_{t}^{\nu}=0, \quad P_{a}^{b}=\delta_{a}^{b} \quad \text { and } \quad P_{a}^{t}=g X_{a} \times D_{t}^{-1} .
$$

This shows that $R$ does not contain a variation $\frac{\delta}{\delta \omega}$, cf. formula (1.19) in [55] (with $\mu=t$ ). The second round bracket is just the decomposition of $\frac{1}{2} A_{\rho} \times A_{\lambda}$ in the reduction.

\subsection{Construction in the matrix model}

Alternatively, we may take the first path and construct the map $\mathcal{T}_{g}$ directly for the matrix model, following the strategy of $[49,53]$. To this end, we implement the Lorenz gauge constraint $\dot{\omega}=0$ by adding to the matrix model Lagrangian $(2.28)^{11}$

$$
\mathcal{L}=\frac{1}{2}\left(D_{t} X_{a}\right)^{2}-\frac{1}{4} g^{2}\left(X_{c} \times X_{d}\right)^{2}-\frac{\mathrm{i}}{2} \theta \cdot\left(D_{t}+g \hat{X} \times\right) \theta
$$

with $\hat{X}:=\gamma^{a} X_{a}$ a "gauge-fixing term"

$$
\mathcal{L}^{\prime}=-\frac{1}{2 \xi} \dot{\omega}^{2}+\bar{C} \cdot \partial_{t} D_{t} C
$$

with a real parameter $\xi$ and ghost matrices $C$ and $\bar{C}$. Taking the limit $\xi \rightarrow 0$ puts the theory on the gauge hypersurface.

We aim to directly derive a coupling flow operator $R$ as in (3.5) for the matrix model, which will govern the infinitesimal change in the coupling $g$ for the quantum correlator of an arbitrary bosonic matrix functional $F[X, \omega]$. Keeping in mind the $g$-dependence of the functional integral weight $\mathrm{e}^{\mathrm{i} \int\left(\mathcal{L}+\mathcal{L}^{\prime}\right)}$, we compute (suppressing the subscript in $\langle\cdots\rangle_{g}$ )

$$
\begin{aligned}
\partial_{g}\langle F\rangle & =\left\langle\partial_{g} F+F \partial_{g} \int \mathrm{i}\left(\mathcal{L}+\mathcal{L}^{\prime}\right)\right\rangle \\
& =\left\langle\partial_{g} F+F \mathrm{i} \int\left[D_{t} X_{a} \cdot \omega \times X_{a}-\frac{1}{2} g\left(X_{c} \times X_{d}\right)^{2}-\frac{\mathrm{i}}{2} \theta \cdot(\omega+\hat{X}) \times \theta+\bar{C} \cdot \partial_{t}(\omega \times C)\right]\right. \\
& =\left\langle\partial_{g} F+F \mathrm{i}\left[\delta_{\alpha} \Delta_{\alpha}+\mathrm{i} q \int \theta \cdot(\omega+\hat{X}) \times \theta+\int \bar{C} \cdot \partial_{t}(\omega \times C)\right]\right\rangle
\end{aligned}
$$

where

$$
\Delta_{\alpha}=-\frac{1}{r} \int \mathrm{d} t\left(\gamma^{d} \theta\right)_{\alpha} \cdot \omega \times X_{d}+\frac{1}{2 r} \int \mathrm{d} t\left(\gamma^{c d} \theta\right)_{\alpha} \cdot X_{c} \times X_{d} .
$$

With the supersymmetry transformations

$$
\delta_{\alpha} \omega=-\mathrm{i} \theta_{\alpha}, \quad \delta_{\alpha} X_{a}=-\mathrm{i}\left(\theta \gamma_{a}\right)_{\alpha}, \quad \delta_{\alpha} \theta_{\beta}=-\gamma_{\alpha \beta}^{d} D_{t} X_{d}-\frac{g}{2} \gamma_{\alpha \beta}^{c d} X_{c} \times X_{d}
$$

\footnotetext{
${ }^{11}$ Here and below, the $\cdot$ denotes a contraction in color space, i.e. $P \cdot Q:=\delta^{A B} P^{A} Q^{B}$.
} 
one confirms that indeed

$$
\delta_{\alpha} \Delta_{\alpha}=\int \mathrm{d} t\left[D_{t} X_{a} \cdot \omega \times X_{a}-\frac{1}{2} g\left(X_{c} \times X_{d}\right)^{2}-\mathrm{i} \frac{D-1}{r} \theta \cdot(\omega+\hat{X}) \times \theta\right] .
$$

Therefore, $\delta_{\alpha} \Delta_{\alpha}$ in (3.15) reproduces $\partial_{g} \int \mathcal{L}$ but with a mismatch in the coefficient of the Majorana term, which thus still appears there but with a coefficient

$$
q=\frac{D-1}{r}-\frac{1}{2}=\frac{1}{r} \quad \text { for } \quad D=3,4,6,10 .
$$

It is noteworthy that for a temporal gauge this mismatch is absent,

$$
A_{0}=0 \Rightarrow \omega=0 \text { and } D_{t}=\partial_{t} \Rightarrow \delta_{\alpha}\left(\left.\Delta_{\alpha}\right|_{\omega=0}\right)=\partial_{g} \int \mathcal{L},
$$

since effectively $D \rightarrow D-1$ and the ghosts decouple.

Next, we employ the broken supersymmetric Ward identity $\left\langle\delta_{\alpha} Y\right\rangle=-\mathrm{i}\left\langle\left(\delta_{\alpha} \int \mathcal{L}^{\prime}\right) Y\right\rangle$ together with

$$
\delta_{\alpha} \int \mathcal{L}^{\prime}=-s \delta_{\alpha} \Delta_{\mathrm{gh}} \quad \text { for } \quad \Delta_{\mathrm{gh}}=\int \bar{C} \dot{\omega}
$$

and the Slavnov variations

$$
s \omega=D_{t} C, \quad s X_{a}=g X_{a} \times C, \quad s \theta=g \theta \times C, \quad s C=-\frac{g}{2} C \times C, \quad s \bar{C}=\frac{1}{\xi} \dot{\omega}
$$

to rewrite

$$
\begin{aligned}
\partial_{g}\langle F\rangle & =\left\langle\partial_{g} F+\mathrm{i} \Delta_{\alpha} \delta_{\alpha} F\right\rangle+\left\langle F\left[\Delta_{\alpha} s \delta_{\alpha} \Delta_{\mathrm{gh}}-q \int \theta \cdot(\omega+\hat{X}) \times \theta+\mathrm{i} \int \bar{C} \cdot \partial_{t}(\omega \times C)\right]\right\rangle \\
& =\left\langle\partial_{g} F+\mathrm{i} \Delta_{\alpha} \delta_{\alpha} F-\Delta_{\alpha}\left(\delta_{\alpha} \Delta_{\mathrm{gh}}\right) s F\right\rangle \\
& +\left\langle F\left[\left(s \Delta_{\alpha}\right)\left(\delta_{\alpha} \Delta_{\mathrm{gh}}\right)-q \int \theta \cdot(\omega+\hat{X}) \times \theta-\mathrm{i} \int \dot{\bar{C}} \cdot(\omega \times C)\right]\right\rangle
\end{aligned}
$$

where in the last step we used the BRST Ward identity $\langle s Y\rangle=0$.

For the flow equation (3.5) to hold, the last correlator has to vanish for any bosonic functional $F$. Writing out

$$
s \Delta_{\alpha}=\frac{1}{r} \int(\hat{X} \times \theta)_{\alpha} \cdot \dot{C} \quad \text { and } \quad \delta_{\alpha} \Delta_{\text {gh }}=-\mathrm{i} \int \dot{\bar{C}} \cdot \theta_{\alpha}
$$

and performing the functional integrations over the fermions and the ghosts, this requirement becomes

$$
0 \stackrel{!}{=}-\frac{\mathrm{i}}{r} \int\left(\hat{X}_{\alpha \beta} \times \theta_{\beta}\right) \cdot \dot{C} \int \dot{\bar{C}} \cdot \theta_{\alpha}-q \int\left(\hat{X}_{\alpha \beta} \times \theta_{\beta}\right) \cdot \theta_{\alpha}-q \int\left(\omega \times \theta_{\alpha}\right) \cdot \theta_{\alpha}+\mathrm{i} \int(\omega \times C) \cdot \dot{\bar{C}},
$$

where the contractions stand for the fermionic and ghost propagators

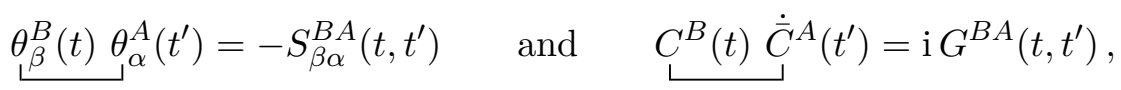


respectively (note the time derivative on $\bar{C}^{A}$ ). With $\partial_{t} G\left(t, t^{\prime}\right)^{B A}=\partial_{t^{\prime}} G^{A B}\left(t^{\prime}, t\right)=: \partial G^{A B}\left(t^{\prime}, t\right)$ the condition (3.25) reads

$$
0 \stackrel{!}{=}-\frac{1}{r} \operatorname{Tr}[(\hat{X} \times S) \partial G]+q \operatorname{Tr}[\hat{X} \times S]+q \operatorname{Tr}[\omega \times S]-\frac{1}{r} \operatorname{Tr}[\omega \times G],
$$

where the trace here refers to spin, color and time altogether. Abbreviating the unit operator by the symbol $\mathbb{1}$, and inserting the useful identities

$$
\partial G=\mathbb{1}-g \omega \times G \quad \text { and } \quad S=G-g G(\hat{X} \times S),
$$

into the first and third term, respectively, we cancel the second and fourth terms (provided $q=\frac{1}{r}$ ) and remain with

$$
0 \stackrel{!}{=} \frac{1}{r} g \operatorname{Tr}[(\hat{X} \times S)(\omega \times G)]-q g \operatorname{Tr}[(\omega \times G)(\hat{X} \times S)]
$$

which indeed holds in the critical dimensions.

We return to (3.23) and integrate out the fermions and ghosts to read off the flow operator

$$
\begin{aligned}
R_{g}= & \mathrm{i} \Delta_{\alpha} \delta_{\alpha}-\Delta_{\alpha}\left(\delta_{\alpha} \Delta_{\mathrm{gh}}\right) s \\
= & \Delta_{\alpha} \int \theta_{\alpha} \cdot \frac{\delta}{\delta \omega}+\Delta_{\alpha} \int\left(\theta \gamma_{a}\right)_{\alpha} \cdot \frac{\delta}{\delta X_{a}} \\
& -\mathrm{i} \Delta_{\alpha} \int \theta_{\alpha} \cdot \dot{\bar{C}} \int D_{t} C \cdot \frac{\delta}{\delta \omega}-\mathrm{i} \Delta_{\alpha} \int \theta_{\alpha} \cdot \dot{\bar{C}} \int\left(g X_{a} \times C\right) \cdot \frac{\delta}{\delta X_{a}} .
\end{aligned}
$$

Since $D_{t} G=\mathbb{1}$, the two variations w.r.t. $\omega$ (first and third terms) cancel, and we are left with

$$
R_{g}=\Delta_{\alpha} \int \theta_{\beta} \cdot\left[\left(\gamma_{a}\right)_{\beta \alpha} \mathbb{1}+g \delta_{\beta \alpha} G \times X_{a}\right] \cdot \frac{\delta}{\delta X_{a}} .
$$

In the curly brackets we recognize the (dimensionally reduced) non-abelian projector (3.12). Recalling $\Delta_{\alpha}$ from (3.16), inserting the fermion propagator (3.26) and reversing the multiplication order, one again arrives at the flow operator presented in (3.10).

\subsection{The map to third and fourth order}

For the perturbative power series we need the expansion of the propagators,

$$
\begin{aligned}
G & =\varepsilon-g \varepsilon \omega \times \varepsilon+g^{2} \varepsilon \omega \times \varepsilon \omega \times \varepsilon-g^{3} \varepsilon \omega \times \varepsilon \omega \times \varepsilon \omega \times \varepsilon \pm \ldots, \\
S & =\varepsilon-g \varepsilon(\omega+\hat{X}) \times \varepsilon+g^{2} \varepsilon(\omega+\hat{X}) \times \varepsilon(\omega+\hat{X}) \times \varepsilon \mp \ldots,
\end{aligned}
$$

with the free fermion propagator (2.37). Because spin traces vanish for an odd product of gamma matrices (for less than nine factors), the expansion coefficients $\mathrm{R}_{k}$ displayed here carry only even/odd powers of $\omega$ for $k$ being even/odd. This parity extends to the map $\mathcal{T}_{g}$ 
itself. Carrying out the spin traces, one gets

$$
\begin{aligned}
\overleftarrow{\mathrm{R}_{1}}= & -\frac{\overleftarrow{\delta}}{\delta X_{a}} \varepsilon \omega \times X_{a}, \\
\overleftarrow{\mathrm{R}_{2}}= & \frac{\overleftarrow{\delta}}{\delta X_{a}} \varepsilon \omega \times \varepsilon \omega \times X_{a}+\frac{\overleftarrow{\delta}}{\delta X_{a}} \varepsilon X_{b} \times \varepsilon X_{b} \times X_{a}, \\
\overleftarrow{\mathrm{R}_{3}}= & -\frac{\overleftarrow{\delta}}{\delta X_{a}} \varepsilon \omega \times \varepsilon \omega \times \varepsilon \omega \times X_{a}-\frac{\overleftarrow{\delta}}{\delta X_{a}} \varepsilon \omega \times \varepsilon X_{b} \times \varepsilon X_{b} \times X_{a}-\frac{\overleftarrow{\delta}}{\delta X_{a}} \varepsilon X_{b} \times \varepsilon \omega \times \varepsilon X_{b} \times X_{a} \\
& -\frac{\overleftarrow{\delta}}{\delta X_{a}} \varepsilon X_{a} \times \varepsilon X_{b} \times \varepsilon \omega \times X_{b}+\frac{\overleftarrow{\delta}}{\delta X_{a}} \varepsilon X_{b} \times \varepsilon X_{a} \times \varepsilon \omega \times X_{b}-\frac{\overleftarrow{\delta}}{\delta X_{a}} \varepsilon X_{b} \times \varepsilon X_{b} \times \varepsilon \omega \times X_{a} \\
& +\frac{\overleftarrow{\delta}}{\delta X_{a}} X_{a} \times \varepsilon \varepsilon X_{b} \times \varepsilon \omega \times X_{b},
\end{aligned}
$$

and so on. Inserting all these into (3.7), performing the functional derivatives and observing various cancellations, we arrive at

$$
\begin{aligned}
\mathcal{T}_{g} X_{a}= & X_{a}+g \varepsilon \omega \times X_{a}-\frac{1}{2} g^{2} \varepsilon X_{b} \times \varepsilon X_{b} \times X_{a} \\
& +\frac{1}{6} g^{3}\left[2 \varepsilon X_{b} \times \varepsilon \omega \times \varepsilon X_{b} \times X_{a}+2 \varepsilon X_{a} \times \varepsilon X_{b} \times \varepsilon \omega \times X_{b}-2 X_{a} \times \varepsilon \varepsilon X_{b} \times \varepsilon \omega \times X_{b}\right. \\
& \left.\quad-\varepsilon X_{b} \times \varepsilon X_{a} \times \varepsilon \omega \times X_{b}+\varepsilon X_{b} \times \varepsilon X_{b} \times \varepsilon \omega \times X_{a}+\varepsilon\left(\varepsilon X_{b} \times X_{a}\right) \times\left(\varepsilon \omega \times X_{b}\right)\right] \\
& +O\left(g^{4}\right) .
\end{aligned}
$$

For the practitioner's convenience we spell this out with our shorthand notation fully expanded,

$$
\begin{aligned}
\mathcal{T}_{g} X_{a}^{A}(t)= & X_{a}^{A}(t)+g f^{A B C} \int \mathrm{d} s \varepsilon(t-s) \omega^{B} X_{a}^{C}(s) \\
& -\frac{1}{2} g^{2} f^{A B C} f^{C D E} \int \mathrm{d} s \mathrm{~d} u \varepsilon(t-s) X_{b}^{B}(s) \varepsilon(s-u) X_{b}^{D} X_{a}^{E}(u) \\
& +\frac{1}{6} g^{3} f^{A B C} f^{C D E} f^{E M N} \int \mathrm{d} s \mathrm{~d} u \mathrm{~d} v \varepsilon(t-s) X_{b}^{B}(s) \varepsilon(s-u)[ \\
& \left.2 \omega^{D}(u) \varepsilon(u-v) X_{b}^{M} X_{a}^{N}(v)-X_{a}^{D}(u) \varepsilon(u-v) \omega^{M} X_{b}^{N}(v)+X_{b}^{D}(u) \varepsilon(u-v) \omega^{M} X_{a}^{N}(v)\right] \\
& +\frac{1}{3} g^{3} f^{A B C} f^{C D E} f^{E M N} \int \mathrm{d} s \mathrm{~d} u \mathrm{~d} v \varepsilon(t-s)\left[X_{a}^{A}(s)-X_{a}^{A}(t)\right] \varepsilon(s-u) X_{b}^{D}(u) \varepsilon(u-v) \omega^{M} X_{b}^{N}(v) \\
& +\frac{1}{6} g^{3} f^{A B C} f^{B D E} f^{C M N} \int \mathrm{d} s \mathrm{~d} u \mathrm{~d} v \varepsilon(t-s)\left[\varepsilon(s-u) X_{b}^{D} X_{a}^{E}(u)\right]\left[\varepsilon(s-v) \omega^{M} X_{b}^{N}(v)\right] \\
& +\mathcal{O}\left(g^{4}\right) .
\end{aligned}
$$

As a check, beyond $\mathcal{O}(g)$ all terms linear in $X_{a}^{A}$ (and thus of maximal power in $\omega^{A}$ ) cancel out, a feature that can be proven to hold in general. Also, in the temporal gauge $\omega^{A}=0$ the map drastically simplifies and admits only even powers in $g$ at least up to the order considered. Moreover, to the order displayed here all terms share the "linear tree" topology of the flow operator, except for the last term in $O\left(g^{3}\right)$, which is the first "branched tree". We have checked that this result is consistent with the final result of [20]. It is straightforward though tedious to extend the above computation to higher orders. Equivalently, the $\mathcal{O}\left(g^{4}\right)$ result 
can be read off by dimensionally reducing the result of [20], but we refrain here from spelling out this formula with non-vanishing $\omega$ because it is rather lengthy and not very illuminating.

However, the result simplifies greatly in the temporal gauge $\omega=0$. Furthermore, given our transcription rule (2.27), it is straightforward to write it down right away with the APD brackets (2.21). Suppressing the common argument $\boldsymbol{\sigma}$, we arrive at

$$
\begin{aligned}
\mathcal{T}_{g} X_{a}(t)= & X_{a}(t)-\frac{1}{2} g^{2} \int \mathrm{d} s \mathrm{~d} u \varepsilon(t-s) \varepsilon(s-u)\left\{X_{b}(s),\left\{X_{b}(u), X_{a}(u)\right\}\right\} \\
+ & \frac{1}{8} g^{4} \int \mathrm{d} s \mathrm{~d} u \mathrm{~d} v \mathrm{~d} w \varepsilon(t-s) \varepsilon(s-u) \varepsilon(u-v) \varepsilon(v-w)[ \\
& 6\left\{X_{b}(s),\left\{X_{c}(u),\left\{X_{[a}(v),\left\{X_{b}(w), X_{c]}(w)\right\}\right\}\right\}\right\} \\
+ & 2\left\{X_{b}(s),\left\{X_{[b}(u),\left\{X_{|c|}(v),\left\{X_{a]}(w), X_{c}(w)\right\}\right\}\right\}\right\} \\
+ & \left.2\left\{X_{a}(s)-X_{a}(t),\left\{X_{b}(u),\left\{X_{c}(v),\left\{X_{b}(w), X_{c}(w)\right\}\right\}\right\}\right\}\right] \\
+ & \frac{1}{8} g^{4} \int \mathrm{d} s \mathrm{~d} u \mathrm{~d} v \mathrm{~d} w \varepsilon(t-s) \varepsilon(s-u) \varepsilon(s-v) \varepsilon(v-w) \times \\
& \left\{\left\{X_{a}(u), X_{b}(u)\right\},\left\{X_{c}(v),\left\{X_{b}(w), X_{c}(w)\right\}\right\}\right\}+\mathcal{O}\left(g^{6}\right) .
\end{aligned}
$$

This expression is perfectly well-defined for well-behaved functions $X_{a}(t, \boldsymbol{\sigma})$, whence the $N \rightarrow \infty$ limit of (3.35) is equally well-defined. At higher orders we will encounter more nested APD brackets, but the expansion stays well-defined to arbitrary order. It is noteworthy that, while (3.35) contains both even and odd powers in $g$, the expansion (3.36) with the temporal gauge $\omega=0$ contains only even powers in $g$. This can only change in higher orders (starting with $R_{7}$ in (3.6), to be completely precise) when we encounter $\gamma$-traces such as $\operatorname{tr}\left(\gamma^{a_{1}} \cdots \gamma^{a_{9}}\right)=16 \epsilon^{a_{1} \cdots a_{9}}$.

Finally, as an independent check, in the appendix we also demonstrate that the "freeaction condition"

$$
\frac{1}{2}\left(\partial_{t} \mathcal{T}_{g} X_{a}\right)^{2} \stackrel{!}{=} \frac{1}{2}\left(D_{t} X_{a}\right)^{2}-\frac{1}{4} g^{2}\left(X_{b} \times X_{c}\right)^{2}+\text { total derivative }
$$

as well as the "determinant matching condition"12

$$
\operatorname{Tr} \log \left(\frac{\delta \mathcal{T}_{g} X}{\delta X}\right) \stackrel{!}{=} \frac{1}{2} \operatorname{Tr} \log \left(D_{t}+g \hat{X} \times\right)+\operatorname{Tr} \log \partial_{t} D_{t}-\frac{D}{2} \operatorname{Tr} \log \partial_{t}^{2}
$$

for the Jacobian, Matthews-Salam-Seiler [46, 47] and Faddeev-Popov [44] determinants are both fulfilled up to and including $O\left(g^{3}\right)$ by the maps (3.34) and (3.36), provided

$$
D-2 \stackrel{!}{=} \frac{r}{2}
$$

\footnotetext{
${ }^{12}$ For the temporal gauge the last two terms on the r.h.s. are replaced by $\left[\operatorname{Tr} \log D_{t}-\frac{D-1}{2} \operatorname{Tr} \log \partial_{t}^{2}\right]$, but the cancellations remain the same, of course.
} 
as happens to be the case for the critical dimensions $D=3,4,6$ and 10. As already mentioned, the determinants are more subtle in the APD gauge theory directly: like for the APD integral kernel (2.39) we can in each APD bracket (2.21) separate the two $\boldsymbol{\sigma}$ arguments by inserting $\delta$-functions together with integrals over $\boldsymbol{\sigma}$ variables. When expanded, the Jacobian of the map $\mathcal{T}_{g}$ then contains exactly the same divergent factor $\delta(\boldsymbol{\sigma}, \boldsymbol{\sigma})$ that we encountered in the expansion of $\Delta_{\text {MSS }}$ and which can thus be dropped for the same reason.

The results of this section should be considered as a generalization of the polynomial map that obtains in supersymmetric quantum mechanics (see e.g. [49]), where the perturbative expansion terminates after the first step and gives rise to a closed expression (see also $[56,57]$ for attempts to find polynomial maps in higher dimensions). Such a feature cannot be expected for the APD gauge theory or matrix model. However, our expressions (3.35) and (3.36) are almost as good, because they can be obtained from a universal formula for $\mathcal{T}_{g}$ in terms of a path-ordered exponential [24]. This formula furnishes an algorithmic procedure to work out the expansion of $\mathcal{T}_{g}$ systematically to any given order in $g$, a calculation that can be automated and implemented on a computer. Again the result will be much simpler with the temporal gauge $\omega=0$. On the technical side it is worth emphasizing that because of (2.34) the differences between the axial and the Lorenz gauge choices almost disappear in one dimension, together with the considerable complications accompanying gauge choices different from the Lorenz gauge in higher dimensions.

Let us, however, alert readers to a technical obstacle that must be overcome before a perturbative evaluation of correlators analogous to [23] can be set up. In the limit of vanishing gauge coupling the measure in (2.31) becomes ultralocal in $\boldsymbol{\sigma}$, with free propagators

$$
\left\langle X^{a}\left(t_{1}, \boldsymbol{\sigma}_{1}\right) X^{b}\left(t_{2}, \boldsymbol{\sigma}_{2}\right)\right\rangle_{0}=\delta^{a b} C\left(t_{1}-t_{2}\right) \delta\left(\boldsymbol{\sigma}_{1}, \boldsymbol{\sigma}_{2}\right)
$$

where $C(t)$ is the free scalar propagator in one time dimension. The reason is that, in the Lagrangian (2.22), the spatial derivatives reside in the interaction term and are not part of the free measure. Consequently, one would have to sum an infinite number of terms to expose the full non-local structure of the theory. As we remarked in the introduction and summary, this problem closely resembles the one of explaining the emergence of spatial structure from the ultralocal BKL limit in quantum cosmology [25]. In both cases, the 'small tension limit' is analogous to the one in the theory of elastic media when neighboring spatial points become decoupled. Let us finally note that via the map $\mathcal{T}_{g}$ the continuous spectrum of the interacting theory is almost self-evident from that of the free theory.

\section{The Jacobian has a non-zero radius of convergence}

One main difference between the present approach and more conventional perturbative expansions of the path integral is that the series expansion for $\mathcal{T}_{g}$ has better convergence properties (here we are not referring to UV divergences, but to the non-summability of what would be the renormalized perturbation expansion in higher dimensions). That the convergence properties should be better was already anticipated in [49] but never actually proven. Here we present further evidence for this conjecture by showing that with suitable technical assumptions the Jacobian of the map admits a non-zero radius of convergence 
when expanded around $g=0$ in the complex $g$ plane. This we can do by exploiting the equality (3.2) of the Jacobian with (the product of) the fermionic determinants. Since we are actually only interested in the statement for the temporal gauge let us therefore set $\omega=0$ for which $\Delta_{\text {FP }}$ is trivial, and consider the MSS determinant (2.35). To this aim we expand the logarithm of $\Delta_{\text {MSS }}$ and make use of the triangle inequality,

$$
\left|\log \operatorname{det}^{1 / 2}(\mathbb{1}+g K)\right|=\frac{1}{2}\left|\sum_{n=1}^{\infty} \frac{(-1)^{n-1}}{n} g^{n} \operatorname{Tr} K^{n}\right| \leq \frac{1}{2} \sum_{n=1}^{\infty} \frac{|g|^{n}}{n}\left|\operatorname{Tr} K^{n}\right|
$$

where the kernel $K$ is defined in (2.36). Let us have a look at the individual terms: we have

$$
\begin{array}{r}
\operatorname{Tr} K^{n}=\int \mathrm{d} t_{1} \cdots \int \mathrm{d} t_{n} \varepsilon\left(t_{1}-t_{2}\right) \cdots \varepsilon\left(t_{n}-t_{1}\right) \operatorname{tr}\left(\gamma^{a_{1}} \cdots \gamma^{a_{n}}\right) \times \\
\times \operatorname{tr}\left(T^{A_{1}} \cdots T^{A_{n}}\right) X_{a_{1}}^{A_{1}}\left(t_{1}\right) \cdots X_{a_{n}}^{A_{n}}\left(t_{n}\right)
\end{array}
$$

where $T^{A}$ are the $\mathrm{SU}(N)$ generators in the adjoint representation. We can now derive an upper bound on the absolute value of this expression by using $|\varepsilon(t)| \leq \frac{1}{2}$, together with

$$
\left|\operatorname{tr}\left(\gamma^{a_{1}} \cdots \gamma^{a_{n}}\right)\right| \leq r \quad \text { and } \quad\left|\operatorname{tr}\left(T^{A_{1}} \cdots T^{A_{n}}\right)\right| \leq c^{n}
$$

where $c \equiv c_{N}$ is an $N$-dependent positive constant. Furthermore introducing the $\mathrm{L}^{1}$-norm

$$
\|\mathbf{X}\|_{1}:=\sum_{a, A} \int \mathrm{d} t\left|X_{a}^{A}(t)\right|
$$

we can majorize the individual terms to obtain

$$
(4.1) \leq \frac{r}{2} \sum_{n=1}^{\infty}\left(\frac{c}{2}\right)^{n} \frac{|g|^{n}}{n}\|\mathbf{X}\|_{1}^{n}
$$

This series converges for $|g|<2 c^{-1}\|\mathbf{X}\|_{1}^{-1}$. Consequently if we constrain the functions $X_{a}^{A}(t)$ to belong each to the Lebesgue space $\mathrm{L}^{1}(\mathbb{R})$, the series always has an ( $X$-dependent) non-zero radius of convergence.

While the fact that the Jacobian has a non-zero radius of convergence as a function of $g$ does not imply that the map itself has this property, it strongly constrains the series expansion for $\mathcal{T}_{g}$, regardless of the precise form of the functions $X_{a}^{A}(t)$. The main reason that makes the argument work is that, unlike for higher-dimensional Yang-Mills theories, the supersymmetric matrix model has no UV divergences which would necessitate infinite subtractions (as in [47]). With appropriate UV and IR regularizations the above statements remain valid for supersymmetric Yang-Mills theories in higher dimensions, at least with the axial gauge choice (for which, however, $\mathcal{T}_{g}$ is considerably more complicated than for the Lorenz gauge $[20,21])$. So in that case both regulators are necessary for the MSS determinant to make sense in a more rigorous context. 


\section{Outlook}

We hope that the present investigations will open some new and so far unexplored avenues for addressing several outstanding key problems of supermembrane and matrix theory. Among the topics for future investigation we have already highlighted two of these, namely the question of quantum target-space Lorentz invariance, and the problem of computing physically relevant correlations functions. Here our approach provides a perturbative expansion scheme of a type that has not been available in the literature so far. Finally we note that our methods may also turn out to be applicable to matrix string theory [58, 59], which corresponds to the reduction of maximally extended super-Yang-Mills theory to two spacetime dimensions.

\section{Acknowledgments}

We thank Hannes Malcha for help in matching (3.36) with the dimensional reduction of the $\mathcal{O}\left(g^{4}\right)$ result of [20], and Daniele Dorigoni and Jan Plefka for discussions. We are also grateful to the referee for constructive criticism of an earlier version of this paper.

\section{A Tests}

\section{A.1 Free action test}

Writing

$$
\mathcal{T}_{g} X_{a}=X_{a}+g \mathrm{~T}_{1} X_{a}+g^{2} \mathrm{~T}_{2} X_{a}+g^{3} \mathrm{~T}_{3} X_{a}+O\left(g^{4}\right)
$$

we read off from (3.34) the concrete expressions for $\mathrm{T}_{k}$. The free-action condition (3.37) then breaks up into

$$
\begin{gathered}
\dot{X}_{a} \cdot\left(\mathrm{T}_{1} X_{a}\right)^{\cdot} \stackrel{!}{=} \dot{X}_{a} \cdot\left(\omega \times X_{a}\right), \\
\frac{1}{2}\left(\mathrm{~T}_{1} X_{a}\right)^{\cdot} \cdot\left(\mathrm{T}_{1} X_{a}\right)^{\cdot}+\dot{X}_{a} \cdot\left(\mathrm{T}_{2} X_{a}\right)^{\cdot} \stackrel{!}{=} \frac{1}{2}\left(\omega \times X_{a}\right)^{2}-\frac{1}{4}\left(X_{a} \times X_{b}\right)^{2}, \\
\left(\mathrm{~T}_{1} X_{a}\right)^{\cdot} \cdot\left(\mathrm{T}_{2} X_{a}\right)^{\cdot}+\dot{X}_{a} \cdot\left(\mathrm{T}_{3} X_{a}\right)^{\cdot} \stackrel{!}{=} 0
\end{gathered}
$$

modulo total derivatives in $t$.

The first condition is fulfilled since $\left(\mathrm{T}_{1} X_{a}\right)^{\cdot}=\omega \times X_{a}$. This also matches the first terms on either side of the second condition. Its remainder is also fulfilled because

$$
\begin{aligned}
\dot{X}_{a} \cdot\left(\mathrm{T}_{2} X_{a}\right)^{\cdot} & =-\frac{1}{2} \dot{X}_{a} \cdot\left(X_{b} \times \varepsilon X_{b} \times X_{a}\right)=-\frac{1}{2}\left(\dot{X}_{a} \times X_{b}\right) \cdot \varepsilon\left(X_{b} \times X_{a}\right) \\
& =-\frac{1}{4}\left(X_{a} \times X_{b}\right)^{\cdot} \cdot \varepsilon\left(X_{b} \times X_{a}\right)=-\frac{1}{4}\left(X_{a} \times X_{b}\right)^{2}+\partial_{t}(\ldots) .
\end{aligned}
$$


The third condition is more involved. The left-hand side reads (suppressing total derivatives)

$$
\begin{aligned}
- & \frac{1}{2}\left(\omega \times X_{a}\right) \cdot X_{b} \times \varepsilon\left(X_{b} \times X_{a}\right)+\frac{1}{6} \dot{X}_{a} \cdot\left(\varepsilon X_{b} \times X_{a}\right) \times\left(\varepsilon \omega \times X_{b}\right) \\
+ & \frac{1}{3} \dot{X}_{a} \cdot\left\{X_{b} \times \varepsilon \omega \times\left(X_{b} \times X_{a}\right)+X_{a} \times \varepsilon X_{b} \times \varepsilon\left(\omega \times X_{b}\right)-\partial_{t}\left\{\left(X_{a} \times \varepsilon \varepsilon X_{b} \times \varepsilon\left(\omega \times X_{b}\right)\right\}\right.\right. \\
& \left.\quad-\frac{1}{2} X_{b} \times \varepsilon X_{a} \times \varepsilon\left(\omega \times X_{b}\right)+\frac{1}{2} X_{b} \times \varepsilon X_{b} \times \varepsilon\left(\omega \times X_{a}\right)\right\} \\
= & -\frac{1}{2}\left(\omega \times X_{a}\right) \times X_{b} \cdot \varepsilon\left(X_{b} \times X_{a}\right)-\frac{1}{6} X_{a} \cdot \partial_{t}\left\{\left(\varepsilon X_{b} \times X_{a}\right) \times\left(\varepsilon \omega \times X_{b}\right)\right\} \\
& +\frac{1}{3} \dot{X}_{a} \times\left\{X_{b} \cdot \varepsilon \omega \times \varepsilon\left(X_{b} \times X_{a}\right)-\dot{X}_{a} \cdot \varepsilon \varepsilon X_{b} \times \varepsilon\left(\omega \times X_{b}\right)-X_{b} \cdot \varepsilon X_{[a} \times \varepsilon\left(\omega \times X_{b]}\right)\right\} \\
= & -\frac{1}{2}\left(\omega \times X_{a}\right) \times X_{b} \cdot \varepsilon\left(X_{b} \times X_{a}\right)-\frac{1}{6} X_{a} \cdot\left(X_{b} \times X_{a}\right) \times \varepsilon\left(\omega \times X_{b}\right)+\frac{1}{6} X_{a} \cdot\left(\omega \times X_{b}\right) \times \varepsilon\left(X_{b} \times X_{a}\right) \\
& +\frac{1}{6}\left(X_{a} \times X_{b}\right)^{\cdot} \cdot \varepsilon \omega \times \varepsilon\left(X_{b} \times X_{a}\right)-\frac{1}{6}\left(X_{a} \times X_{b}\right)^{\cdot} \cdot \varepsilon X_{a} \times \varepsilon\left(\omega \times X_{b}\right) \\
= & -\frac{1}{2}\left(\omega \times X_{a}\right) \times X_{b} \cdot \varepsilon\left(X_{b} \times X_{a}\right)-\frac{1}{6} X_{a} \times\left(X_{b} \times X_{a}\right) \cdot \varepsilon\left(\omega \times X_{b}\right)+\frac{1}{6} X_{a} \times\left(\omega \times X_{b}\right) \cdot \varepsilon\left(X_{b} \times X_{a}\right) \\
& -\frac{1}{6}\left(X_{a} \times X_{b}\right) \cdot \omega \times \varepsilon\left(X_{b} \times X_{a}\right)+\frac{1}{6}\left(X_{a} \times X_{b}\right) \cdot X_{a} \times \varepsilon\left(\omega \times X_{b}\right) \\
= & -\frac{1}{6}\left\{3\left(\omega \times X_{a}\right) \times X_{b}-X_{a} \times\left(\omega \times X_{b}\right)+\left(X_{a} \times X_{b}\right) \times \omega\right\} \cdot \varepsilon\left(X_{b} \times X_{a}\right) \\
= & -\frac{1}{6}\left\{3\left(\omega \times X_{[a}\right) \times X_{b]}+\left(\omega \times X_{[b}\right) \times X_{a]}+\left(X_{a} \times X_{b}\right) \times \omega\right\} \cdot \varepsilon\left(X_{b} \times X_{a}\right) \\
= & -\frac{1}{6}\left\{\left(\omega \times X_{a}\right) \times X_{b}+\left(X_{b} \times \omega\right) \times X_{a}+\left(X_{a} \times X_{b}\right) \times \omega\right\} \cdot \varepsilon\left(X_{b} \times X_{a}\right)=0,
\end{aligned}
$$

where in the last line the Jacobi identity was applied. Several times we employed partial integration and $\partial_{t} \varepsilon=\mathbb{1}$ as well as $A \cdot(B \times C)=(A \times B) \cdot C$ and the complete antisymmetry of the structure constants, $A \times B=-B \times A$. Furthermore, for the first equality we cancelled part of the $\partial_{t}$ term with the term preceding it, for the second equality we dropped a term $\sim \dot{X}_{a} \times \dot{X}_{a}=0$, for the fourth equality the second and fifth terms cancelled, and for the fifth equality the index antisymmetry in the final factor $X_{b} \times X_{a}$ was used. We note that the value $D$ of the spacetime dimension played no role here.

\section{A.2 Determinant matching test}

Since the determinants match in the free theory, it suffices to bring their logarithms to a form

$$
\log \operatorname{det} \Delta(g)=\log \operatorname{det} \Delta(0)+\operatorname{Tr} \log (\mathbb{1}+M(g))=\mathrm{const}+\operatorname{Tr} M-\frac{1}{2} \operatorname{Tr} M^{2}+\frac{1}{3} \operatorname{Tr} M^{3}+O\left(g^{4}\right)
$$

since $M(g)$ is of order $g$, and to compare the expressions in the orders $g, g^{2}$ and $g^{3}$ of the perturbative expansion. The Tr symbol refers to a trace in position, color and spinor space, while below we reserve the tr symbol for the trace in position and color space only, after having explicitly performed the gamma traces.

For the Faddeev-Popov determinant we have

$$
\Delta=\partial_{t} D_{t}=\partial_{t}\left(\partial_{t}+g \omega \times\right) \Rightarrow M=g \partial_{t}^{-1} \omega \times=g \varepsilon \omega \times
$$


which, since there are no spin degreees of freedom, leads to

$$
\operatorname{tr} \log (\mathbb{1}+M(g))=g \operatorname{tr}(\omega \times \varepsilon)-\frac{1}{2} g^{2} \operatorname{tr}(\omega \times \varepsilon \omega \times \varepsilon)+\frac{1}{3} g^{3} \operatorname{tr}(\omega \times \varepsilon \omega \times \varepsilon \omega \times \varepsilon)+O\left(g^{4}\right) .
$$

The Matthews-Salam-Seiler determinant produces

$$
\Delta=D_{t}+g \hat{X} \times=\partial_{t}+g(\omega+\hat{X}) \times \Rightarrow M=g \varepsilon(\omega+\hat{X}) \times
$$

which, with a factor of $\frac{1}{2}$ from the Majorana property, yields

$$
\begin{aligned}
\frac{1}{2} \operatorname{Tr} \log (\mathbb{1}+M(g))= & \frac{r}{2} g \operatorname{tr}(\omega \times \varepsilon)-\frac{r}{4} g^{2} \operatorname{tr}\left(\omega \times \varepsilon \omega \times \varepsilon+X_{a} \times \varepsilon X_{a} \times \varepsilon\right) \\
& +\frac{r}{6} g^{3} \operatorname{tr}\left(\omega \times \varepsilon \omega \times \varepsilon \omega \times \varepsilon+3 X_{a} \times \varepsilon X_{a} \times \varepsilon \omega \times \varepsilon\right)+O\left(g^{4}\right),
\end{aligned}
$$

where only even powers of $\hat{X}$ survived the spin trace, which produces a factor $r$ for the dimensionality of the spinor representation. Each of the trace terms can be represented by a loop diagram, with bosonic propagators $\varepsilon$ and external "legs" $\omega$ or $X_{a}$. Because $\varepsilon(t-t)=\varepsilon(0)=0$, single-leg loops vanish, and we only have to consider the orders $g^{2}$ and $g^{3}$ in the matching.

Finally, considering the Jacobian of $\mathcal{T}_{g}$, we must in each tree of the expression (3.34) "differentiate away" one "leaf" $X$ in all possible ways. This results in an expression of the form

$$
\frac{\delta \mathcal{T}_{g} X_{a}^{A}(t)}{\delta X_{b}^{B}\left(t^{\prime}\right)}=\delta^{A B} \delta_{a b} \delta\left(t-t^{\prime}\right)+\left(g M_{1}+g^{2} M_{2}+g^{3} M_{3}+O\left(g^{4}\right)\right)_{a b}^{A B}\left(t, t^{\prime}\right),
$$

which can be viewed as a string starting from the tree root and ending at the cut leaf location, possibly with branches attached to it. Inserting this expansion into (A.5) we find

$$
\log \operatorname{det}\left(\frac{\delta \mathcal{T}_{g} X}{\delta X}\right)=\mathrm{const}+g \operatorname{Tr} M_{1}+g^{2}\left(\operatorname{Tr} M_{2}-\frac{1}{2} \operatorname{Tr} M_{1}^{2}\right)+g^{3}\left(\operatorname{Tr} M_{3}-\operatorname{Tr} M_{1} M_{2}+\frac{1}{3} \operatorname{Tr} M_{1}^{3}\right) .
$$

Under each trace we glue together the strings in the product and then short-circuit the total string by identifying the end points and summing over the corresponding indices (including integration over time). As a result we collect

$$
\begin{aligned}
\operatorname{Tr} M_{2} & =-\frac{1}{2}(D-2) \operatorname{tr}\left(X_{a} \times \varepsilon X_{a} \times \varepsilon\right), \\
-\frac{1}{2} \operatorname{Tr} M_{1}^{2} & =-\frac{1}{2}(D-1) \operatorname{tr}(\omega \times \varepsilon \omega \times \varepsilon)
\end{aligned}
$$

and, after several cancellations,

$$
\begin{aligned}
\operatorname{Tr} M_{3}= & \left(\frac{D}{2}-\frac{2}{3}\right) \operatorname{tr}\left(X_{a} \times \varepsilon X_{a} \times \varepsilon \omega \times \varepsilon\right)+\frac{1}{3} \operatorname{tr}\left(\varepsilon X_{a} \times \varepsilon\right) \times \varepsilon \omega \times X_{a} \\
& -\frac{1}{3} \operatorname{tr}\left(\varepsilon \varepsilon X_{a} \times\right) \times \varepsilon \omega \times X_{a}-\frac{1}{3} \operatorname{tr}\left(X_{a} \times \varepsilon X_{a} \times \omega \times \varepsilon \varepsilon\right) \\
= & \left(\frac{D}{2}-1\right) \operatorname{tr}\left(X_{a} \times \varepsilon X_{a} \times \varepsilon \omega \times \varepsilon\right)+\frac{1}{3} \operatorname{tr}\left(\varepsilon X_{a} \times \varepsilon\right) \times \varepsilon \omega \times X_{a}, \\
-\operatorname{Tr} M_{1} M_{2}= & \left(\frac{D}{2}-1\right) \operatorname{tr}\left(X_{a} \times \varepsilon X_{a} \times \varepsilon \omega \times \varepsilon\right), \\
\frac{1}{3} \operatorname{Tr} M_{1}^{3}= & \frac{1}{3}(D-1) \operatorname{tr}(\omega \times \varepsilon \omega \times \varepsilon \omega \times \varepsilon) .
\end{aligned}
$$


In the four contributions to $\operatorname{Tr} M_{3}$, the fourth term is of the same form as the first one because $\omega$ being constant can be moved past $\varepsilon$. The other two contributions are loops with a branch attached. The third term vanishes because the trace is proportional to $\partial_{t}^{-2}(0)$ which gets regularized to zero. Finally, the second term is of the form

$$
f\left(t^{\prime}, t^{\prime \prime}\right) \int \mathrm{d} t \varepsilon\left(t-t^{\prime}\right) \varepsilon\left(t^{\prime}-t\right) \varepsilon\left(t-t^{\prime \prime}\right)=-\frac{1}{4} f\left(t^{\prime}, t^{\prime \prime}\right) \int \mathrm{d} t \varepsilon\left(t-t^{\prime \prime}\right)=0 .
$$

Collecting all remaining contributions, we end up with two 2-leg loops at $O\left(g^{2}\right)$ and two 3-leg loops at $O\left(g^{3}\right)$ :

\begin{tabular}{|l|c|c|c|}
\hline expression & FP & MSS & Jac \\
\hline$g^{2} \operatorname{tr}(\omega \times \varepsilon \omega \times \varepsilon)$ & $-\frac{1}{2}$ & $-\frac{r}{4}$ & $\frac{1}{2}(1-D)$ \\
$g^{2} \operatorname{tr}\left(X_{a} \times \varepsilon X_{a} \times \varepsilon\right)$ & 0 & $-\frac{r}{4}$ & $\frac{1}{2}(2-D)$ \\
\hline$g^{3} \operatorname{tr}(\omega \times \varepsilon \omega \times \varepsilon \omega \times \varepsilon)$ & $\frac{1}{3}$ & $\frac{r}{6}$ & $\frac{1}{3}(D-1)$ \\
$g^{3} \operatorname{tr}\left(X_{a} \times \varepsilon X_{a} \times \varepsilon \omega \times \varepsilon\right)$ & 0 & $3 \frac{r}{6}$ & $D-2$ \\
\hline
\end{tabular}

Here, "FP", "MSS" and "Jac" denote the weight of the individual expressions contributing to the logarithm of the Faddeev-Popov, Matthews-Salam-Seiler and Jacobian determinant, respectively. Fortunately, the sum of the FP and MSS columns agrees with the Jac column provided that again $D-2=\frac{r}{2}$, singling out the critical dimensions once more. This provides a nontrivial check on the expression (3.35) of the Nicolai map, which formally is guaranteed to work out by the construction scheme. Finally, we remark that the matching also works in the temporal gauge, since the Faddeev-Popov determinant becomes trivial but the first and third expression in the table vanish for $\omega=0$ anyway.

Open Access. This article is distributed under the terms of the Creative Commons Attribution License (CC-BY 4.0), which permits any use, distribution and reproduction in any medium, provided the original author(s) and source are credited.

\section{References}

[1] E. Bergshoeff, E. Sezgin and P.K. Townsend, Supermembranes and Eleven-Dimensional Supergravity, Phys. Lett. B 189 (1987) 75 [INSPIRE].

[2] E. Bergshoeff, E. Sezgin and P.K. Townsend, Properties of the Eleven-Dimensional Super Membrane Theory, Annals Phys. 185 (1988) 330 [INSPIRE].

[3] E. Cremmer, B. Julia and J. Scherk, Supergravity Theory in Eleven-Dimensions, Phys. Lett. B 76 (1978) 409 [INSPIRE].

[4] B. de Wit, J. Hoppe and H. Nicolai, On the Quantum Mechanics of Supermembranes, Nucl. Phys. B 305 (1988) 545 [INSPIRE].

[5] J. Goldstone, unpublished.

[6] J. Hoppe, Quantum theory of a massless relativistic surface and a two-dimensional bound state problem, Ph.D. Thesis, MIT (1982). 
[7] J. Hoppe, Quantum theory of a relativistic surface, in Proc. Int. Workshop on Constraint theory and relativistic dynamics, G. Longhi and L. Lusanna eds., World Scientific (1987) [INSPIRE].

[8] E. Witten, Bound states of strings and p-branes, Nucl. Phys. B 460 (1996) 335 [hep-th/9510135] [INSPIRE].

[9] T. Banks, W. Fischler, S.H. Shenker and L. Susskind, M theory as a matrix model: A Conjecture, Phys. Rev. D 55 (1997) 5112 [hep-th/9610043] [INSPIRE].

[10] M.J. Duff, Supermembranes, in Theoretical Advanced Study Institute in Elementary Particle Physics (TASI 96): Fields, Strings, and Duality, (1996) [hep-th/9611203] [INSPIRE].

[11] H. Nicolai and R. Helling, Supermembranes and M(atrix) theory, in ICTP Spring School on Nonperturbative Aspects of String Theory and Supersymmetric Gauge Theories, pp. 29-74 (1998) [hep-th/9809103] [INSPIRE].

[12] B. de Wit, Supermembranes and super matrix models, Lect. Notes Phys. 525 (1999) 97 [hep-th/9902051] [INSPIRE].

[13] B. de Wit, U. Marquard and H. Nicolai, Area Preserving Diffeomorphisms and Supermembrane Lorentz Invariance, Commun. Math. Phys. 128 (1990) 39 [inSPIRE].

[14] K. Ezawa, Y. Matsuo and K. Murakami, Lorentz symmetry of supermembrane in light cone gauge formulation, Prog. Theor. Phys. 98 (1997) 485 [hep-th/9705005] [INSPIRE].

[15] U. Marquard, R. Kaiser and M. Scholl, Lorentz Algebra and Critical Dimension for the Supermembrane, Phys. Lett. B 227 (1989) 234 [INSPIRE].

[16] A. Dasgupta, H. Nicolai and J. Plefka, Vertex operators for the supermembrane, JHEP 05 (2000) 007 [hep-th/0003280] [INSPIRE].

[17] L. Boulton, M.P. García del Moral and A. Restuccia, Existence of a supersymmetric massless ground state of the $\mathrm{SU}(N)$ matrix model globally on its valleys, JHEP 05 (2021) 281 [arXiv:2102.00886] [INSPIRE].

[18] H. Nicolai, On a New Characterization of Scalar Supersymmetric Theories, Phys. Lett. B 89 (1980) 341 [INSPIRE].

[19] H. Nicolai, Supersymmetry and Functional Integration Measures, Nucl. Phys. B 176 (1980) 419 [INSPIRE].

[20] H. Malcha and H. Nicolai, Perturbative linearization of super-Yang-Mills theories in general gauges, JHEP 06 (2021) 001 [arXiv:2104.06017] [INSPIRE].

[21] O. Lechtenfeld and M. Rupprecht, Construction method for the Nicolai map in supersymmetric Yang-Mills theories, Phys. Lett. B 819 (2021) 136413 [arXiv:2104.09654] [InSPIRE].

[22] G. Savvidy, Symplectic and large $N$ gauge theories, in Cargese Summer School Vacuum Structure in Intense Fields, pp. 415-427, 30 July-11 August 1990, Cargese, France (C90-07-30) [INSPIRE].

[23] H. Nicolai and J. Plefka, $N=4$ super-Yang-Mills correlators without anticommuting variables, Phys. Rev. D 101 (2020) 125013 [arXiv:2003.14325] [InSPIRE].

[24] O. Lechtenfeld and M. Rupprecht, Universal form of the Nicolai map, Phys. Rev. D 104 (2021) L021701 [arXiv: 2104.00012] [INSPIRE].

[25] T. Damour, M. Henneaux and H. Nicolai, $E_{10}$ and a 'small tension expansion' of M-theory, Phys. Rev. Lett. 89 (2002) 221601 [hep-th/0207267] [INSPIRE]. 
[26] M.J. Duff, P.S. Howe, T. Inami and K.S. Stelle, Superstrings in D $=10$ from Supermembranes in $D=11$, Phys. Lett. B 191 (1987) 70 [INSPIRE].

[27] E. Witten, String theory dynamics in various dimensions, Nucl. Phys. B 443 (1995) 85 [hep-th/9503124] [INSPIRE].

[28] P.K. Townsend, Four lectures on M-theory, in ICTP Summer School in High-energy Physics and Cosmology, pp. 385-438 (1996) [hep-th/9612121] [INSPIRE].

[29] E. Bergshoeff, E. Sezgin and Y. Tanii, Hamiltonian Formulation of the Supermembrane, Nucl. Phys. B 298 (1988) 187 [inSPIRE].

[30] B. de Wit, M. Lüscher and H. Nicolai, The Supermembrane Is Unstable, Nucl. Phys. B 320 (1989) 135 [INSPIRE].

[31] A.V. Smilga, Super-Yang-Mills quantum mechanics and supermembrane spectrum, in Trieste Conference on Supermembranes and Physics in $2+1$ Dimensions, (1989) [arXiv:1406.5987] [INSPIRE].

[32] L.S. Boulton, M.P. Garcia del Moral, I. Martin and A. Restuccia, On the spectrum of a matrix model for the $D=11$ supermembrane compactified on a torus with nontrivial winding, Class. Quant. Grav. 19 (2002) 2951 [hep-th/0109153] [INSPIRE].

[33] L. Brink, J.H. Schwarz and J. Scherk, Supersymmetric Yang-Mills Theories, Nucl. Phys. B 121 (1977) 77 [INSPIRE].

[34] J. Hoppe, Diff $T^{2}$ and the Curvature of Some Infinite Dimensional Manifolds, Phys. Lett. B 215 (1988) 706 [INSPIRE].

[35] D.B. Fairlie, P. Fletcher and C.K. Zachos, Trigonometric Structure Constants for New Infinite Algebras, Phys. Lett. B 218 (1989) 203 [INSPIRE].

[36] M. Bordemann, E. Meinrenken and M. Schlichenmaier, Toeplitz quantization of Kähler manifolds and $g l(N), N \rightarrow \infty$ limits, Commun. Math. Phys. 165 (1994) 281 [hep-th/9309134] [INSPIRE].

[37] M. Claudson and M.B. Halpern, Supersymmetric Ground State Wave Functions, Nucl. Phys. B 250 (1985) 689 [INSPIRE].

[38] M. Baake, M. Reinicke and V. Rittenberg, Fierz Identities for Real Clifford Algebras and the Number of Supercharges, J. Math. Phys. 26 (1985) 1070 [INSPIRE].

[39] R. Flume, On Quantum Mechanics With Extended Supersymmetry and Nonabelian Gauge Constraints, Annals Phys. 164 (1985) 189 [INSPIRE].

[40] D. Bigatti and L. Susskind, Review of matrix theory, NATO Sci. Ser. C 520 (1999) 277 [hep-th/9712072] [INSPIRE].

[41] T. Banks, TASI lectures on matrix theory, in Theoretical Advanced Study Institute in Elementary Particle Physics (TASI 99): Strings, Branes, and Gravity, pp. 495-542 (1999) [hep-th/9911068] [INSPIRE].

[42] W. Taylor, The M(atrix) model of M-theory, NATO Sci. Ser. C 556 (2000) 91 [hep-th/0002016] [inSPIRE].

[43] D. Friedan and S.H. Shenker, The Analytic Geometry of Two-Dimensional Conformal Field Theory, Nucl. Phys. B 281 (1987) 509 [InSPIRE].

[44] L.D. Faddeev and V.N. Popov, Feynman Diagrams for the Yang-Mills Field, Phys. Lett. B 25 (1967) 29 [INSPIRE]. 
[45] G. 't Hooft, Renormalization of Massless Yang-Mills Fields, Nucl. Phys. B 33 (1971) 173 [INSPIRE].

[46] P.T. Matthews and A. Salam, The Green's functions of quantized fields, Nuovo Cim. 12 (1954) 563 [INSPIRE].

[47] E. Seiler, Schwinger Functions for the Yukawa Model in Two-Dimensions with Space-Time Cutoff, Commun. Math. Phys. 42 (1975) 163 [InSPIRE].

[48] M.B. Green and J.H. Schwarz, Supersymmetrical Dual String Theory. 2. Vertices and Trees, Nucl. Phys. B 198 (1982) 252 [INSPIRE].

[49] H. Nicolai, Supersymmetric functional integration measures, in Supersymmetry, K. Dietz et al. eds., Plenum Press (1984).

[50] R. Flume and O. Lechtenfeld, On the Stochastic Structure of Globally Supersymmetric Field Theories, Phys. Lett. B 135 (1984) 91 [INSPIRE].

[51] K. Dietz and O. Lechtenfeld, Nicolai Maps and Stochastic Observables From a Coupling Constant Flow, Nucl. Phys. B 255 (1985) 149 [inSPIRE].

[52] K. Dietz and O. Lechtenfeld, Ghost free quantization of non-Abelian gauge theories via the Nicolai transformation of their supersymmetric extensions, Nucl. Phys. B 259 (1985) 397 [INSPIRE].

[53] O. Lechtenfeld, Construction of the Nicolai mapping in supersymmetric field theories, Ph.D. Thesis, Bonn University (1984) [INSPIRE].

[54] S. Ananth, H. Nicolai, C. Pandey and S. Pant, Supersymmetric Yang-Mills theories:not quite the usual perspective, J. Phys. A 53 (2020) 17 [arXiv:2001.02768] [INSPIRE].

[55] S. Ananth, O. Lechtenfeld, H. Malcha, H. Nicolai, C. Pandey and S. Pant, Perturbative linearization of supersymmetric Yang-Mills theory, JHEP 10 (2020) 199 [arXiv:2005.12324] [INSPIRE].

[56] O. Lechtenfeld, Stochastic variables in ten-dimensions?, Nucl. Phys. B 274 (1986) 633 [INSPIRE].

[57] V. de Alfaro, S. Fubini and G. Furlan, Stochastic identities in the light cone gauge, Phys. Lett. $B 163$ (1985) 176 [INSPIRE].

[58] L. Motl, Proposals on nonperturbative superstring interactions, hep-th/9701025 [INSPIRE].

[59] R. Dijkgraaf, E.P. Verlinde and H.L. Verlinde, Matrix string theory, Nucl. Phys. B 500 (1997) 43 [hep-th/9703030] [INSPIRE]. 